\title{
Impact of Different Random Initializations on Generalization Performance of Extreme Learning Machine
}

\author{
Xiaofang Zhang1 ${ }^{1}$ Xiaoli Lin ${ }^{1}$, Rana Aamir Raza Ashfaq2 ${ }^{2}$ \\ ${ }^{1}$ Information Engineering Department, City College, Wuhan University of Science and Technology, Wuhan \\ 430083, China. \\ ${ }^{2}$ Department of Computer Science, Bahauddin Zakariya University, Multan, Pakistan. \\ * Corresponding author. Email: aamir@bzu.edu.pk \\ Manuscript submitted September 26, 2017; accepted November 21, 2017. \\ doi: $10.17706 /$ jcp.13.7.805-822
}

\begin{abstract}
The generalization performance of extreme learning machine (ELM) is influenced by the random initializations to input-layer weights and hidden-layer biases. In this paper, we demonstrate this conclusion through testing the classification accuracies of ELMs corresponding to different random initializations. 30 UCI data sets and 24 continuous probability distributions are employed in this experimental study. The final results present the following important and valuable observations and conclusions, i.e., (1) the probability distributions with symmetrical and bell-shaped probability density functions (e.g., Hyperbolic Secant, Student's-t, Laplace and Normal) always bring about the higher training accuracies and easily cause the over-fitting of ELM; (2) ELMs with random input-layer weights and hidden-layer biases chosen from heavy-tailed distributions (e.g., Gamma, Rayleigh and Frechet) have the better generalization performances; and (3) the light-tailed distributions (e.g., Central Chi-Squared, Erlang, F, Gumbel and Logistic) are usually unsuited to initialize the input-layer weights and hidden-layer biases for ELM. All these provide the useful enlightenments for practical applications of ELMs in different fields.
\end{abstract}

Key words: Extreme learning machine, ELM, generalization performance, random initialization.

\section{Introduction}

Extreme learning machine (ELM) [1] is a kind of special single hidden-layer feed-forward neural network (SLFN) in which the input-layer weights and hidden-layer biases are randomly selected and the output-layer weights are analytically determined by solving Moore-Penrose generalized inverse [2] of hidden-layer output matrix. Because of avoiding the time-consuming iterations, ELMs obtains the extremely fast training speed. Meanwhile, the theoretically proof [3] guarantees the universal approximate capability of ELM. The lower computational complexity and acceptable generalization performance makes ELM obtain a wide range of practical applications [4].

However, ELM has an obvious defect, i.e., the instability of predictive results. Although Huang et al. in [1] proved the uniform convergence of ELM for any continuous probability distribution, this defect indeed exists in the practical applications due to the inherent character of data set, e.g., noise and outlier, etc. The researchers have developed some representative works to improve the instability of ELM caused by the random initializations of input-layer weights and hidden-layer biases. Zhu et al. [5] proposed an evolutionary ELM (E-ELM) which uses the differential evolutionary algorithm to select the input weights and hidden biases for ELM. Then, Cao et al. [6] improved E-ELM and developed a self-adaptive evolutionary 
extreme learning machine (SaE-ELM) to optimize the hidden node parameters. Experimental results show SaE-ELM outperforms E-ELM. An optimized extreme learning machine (O-ELM) was designed by Matias et al. [7], which uses three different optimization algorithms to simultaneously optimize the input weights, hidden biases, and regularization factor. Heeswijk et al. [8] proposed two weight initialization schemes, i.e., binary ELM based on $\{0,1\}$-weights and ternary ELM based on $\{-1,0,1\}$-weights, to improve the diversity of neurons in the hidden layer. For binary/ternary ELMs, the necessary optimizations are also required to select the better parameters. Form the above-mentioned description, we can find that E-ELM, SaE-ELM, O-ELM, and binary/ternary ELMs used the different optimization algorithms to tune the initially random input-layer weights and hidden-layer biases. These works confirmed the impact of random initialization on generalization capability of ELM.

Usually, the random numbers obeying uniform distribution are used to initialize the input-layer weights and hidden-layer biases, including the original ELM [1], E-ELM [5], SaE-ELM [6], O-ELM [7], binary/ternary ELMs [8], and other forms of improved ELM [3], [9]-[15]. We rarely find ELMs which initialize the parameters with other probability distributions. The main objective of this paper is to validate the impact of different random initializations on generalization performance of ELM. Specifically, we want to clarify the influence of initializations obeying different continues probability distributions on the training and testing accuracies of ELM. 24 different continuous probability distributions [16] as shown in Table 1 are introduced in our study to initialize the input-layer weights and hidden-layer biases for ELMs. We select 30 UCI classification data sets which involve a wide range of real domains and data characteristics. In our experiment, the 10 -fold cross-validation procedure are repeated 100 times. In every time of 10 -fold cross-validation, different ELMs are trained on the same training sets and evaluated on the same testing set. The training and testing accuracies on given data set are the average values of 100 times of 10 -fold cross-validations. We further use Wilcoxon signed-ranks test and Friedman test [17] to compare the classification performances of different ELMs for 30 data sets. The experimental results and statistical analysis show (1) the symmetrical and bell-shaped probability distributions easily cause the over-fitting of ELM; (2) the heavy-tailed distributions bring about the better generalization performance for ELM; and (3) the light-tailed distributions obtain the worse training and testing accuracies than Uniform distribution. The obtained conclusions from experimental results can instruct users choose the appropriate probability distribution to initialize the necessary parameters of ELM so as to reduce the possibility of instability of ELM caused by the random initializations.

The rest of the paper is organized as follows: In Section 2, we summarize the basic extreme learning machine. In Section 3, we experimentally validate the influence of initializations obeying different probability distributions on generalization performance of ELM. We conclude this work with some remarks in the last Section.

\section{Extreme Learning Machine}

Here, we consider using extreme learning machine (ELM) [12] to deal with the classification problem. Given the training dataset $\left(X_{N \times D}, Y_{N \times C}\right)$ and testing dataset $\left(X_{M \times D}^{\prime}, Y_{M \times C}^{\prime}\right)$ :

$$
\begin{gathered}
X_{N \times D}=\left[\begin{array}{c}
x_{1} \\
x_{2} \\
\vdots \\
x_{N}
\end{array}\right]=\left[\begin{array}{cccc}
x_{11} & x_{12} & \cdots & x_{1 D} \\
x_{21} & x_{22} & \cdots & x_{2 D} \\
\vdots & \vdots & \ddots & \vdots \\
x_{N 1} & x_{N 2} & \cdots & x_{N D}
\end{array}\right], Y_{N \times C}=\left[\begin{array}{c}
y_{1} \\
y_{2} \\
\vdots \\
y_{N}
\end{array}\right]=\left[\begin{array}{cccc}
y_{11} & y_{12} & \cdots & y_{1 C} \\
y_{21} & y_{22} & \cdots & y_{2 C} \\
\vdots & \vdots & \ddots & \vdots \\
y_{N 1} & y_{N 2} & \cdots & y_{N C}
\end{array}\right] \\
X_{M \times D}^{\prime}=\left[\begin{array}{c}
x_{1}^{\prime} \\
x_{2}^{\prime} \\
\vdots \\
x_{M}^{\prime}
\end{array}\right]=\left[\begin{array}{cccc}
x_{11}^{\prime} & x_{12}^{\prime} & \cdots & x_{1 D}^{\prime} \\
x_{21}^{\prime} & x_{22}^{\prime} & \cdots & x_{2 D}^{\prime} \\
\vdots & \vdots & \ddots & \vdots \\
x_{M 1}^{\prime} & x_{M 2}^{\prime} & \cdots & x_{M D}^{\prime}
\end{array}\right], Y_{M \times C}^{\prime}=\left[\begin{array}{c}
y_{1}^{\prime} \\
y_{2}^{\prime} \\
\vdots \\
y_{M}^{\prime}
\end{array}\right]=\left[\begin{array}{cccc}
y_{11}^{\prime} & y_{12}^{\prime} & \cdots & y_{1 C}^{\prime} \\
y_{21}^{\prime} & y_{22}^{\prime} & \cdots & y_{2 C}^{\prime} \\
\vdots & \vdots & \ddots & \vdots \\
y_{M 1}^{\prime} & y_{M 2}^{\prime} & \cdots & y_{M C}^{\prime}
\end{array}\right],
\end{gathered}
$$


where $N$ is the number of training instances, $D$ is the number of input variables, $M$ is the number of testing instances, and $C$ is the number of output variables. For $i$-th training instance $\mathrm{x}_{i}(1 \leq i \leq N)$ which belongs to $c$-th $(1 \leq c \leq C)$ class, $y_{i c}=1$ and $y_{i e r}=0\left(c^{\prime}=1,2, \cdots, C\right.$ and $\left.c^{\prime} \neq c\right) . \mathrm{Y}_{M \times C}^{\prime}$ is unknown and needs to be predicted.

ELM is a single hidden-layer feed-forward neural network (SLFN) and does not require any iterative optimization to input/output weights. ELM determines $\mathrm{Y}_{M \times C}^{\prime}$ as follows:

Table 1.24 Continuous Probability Distributions

\begin{tabular}{|c|c|c|c|}
\hline & Distribution & Density function $p(x)$ & Support interval \\
\hline $\mathrm{D}_{1}$ & Beta & $\frac{x^{\alpha-1}(1-x)^{g-1}}{E(\alpha, \beta)}, \alpha>0, \beta>0$ & $x \in[0,1]$ \\
\hline $\mathrm{D}_{2}$ & Cauchy & $\frac{1}{\pi}\left(\frac{\lambda}{x^{2}+\lambda^{2}}\right), \lambda>0$ & $x \in(-\infty,+\infty)$ \\
\hline $\mathrm{D}_{3}$ & Central Chi-Squared & $\frac{2-\frac{k}{2} x \frac{k}{2}-1-1-\frac{x}{2}}{\Gamma(k / 2)}, k>0$ & $x \in[0,+\infty)$ \\
\hline $\mathrm{D}_{4}$ & Chi & $\frac{2^{1-\frac{k}{2} x^{k-1} e^{-\frac{x^{2}}{2}}}}{\Gamma(k / 2)}, k>0$ & $x \in[0,+\infty)$ \\
\hline $\mathrm{D}_{5}$ & Erlang & $\frac{\lambda^{k} x^{k-1} e^{-\lambda x}}{(k-1) !}, k>0, \lambda>0$ & $x \in[0,+\infty)$ \\
\hline $\begin{array}{l}\mathrm{D}_{6} \\
\mathrm{D}_{\overline{7}}\end{array}$ & $\begin{array}{l}\text { Exponential } \\
\mathrm{F}\end{array}$ & 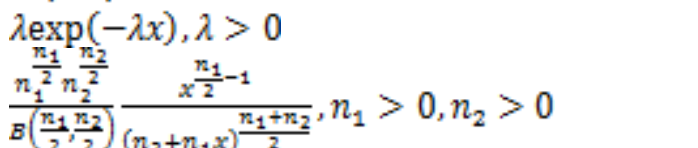 & $\begin{array}{l}x \in[0,+\infty) \\
x \in[0,+\infty)\end{array}$ \\
\hline $\mathrm{D}_{8}$ & Frechet & $\frac{a}{s}\left(\frac{x}{s}\right)^{-1-\alpha} e^{\left(-\frac{x}{s}\right)^{-\alpha}}, \alpha>0, s>0$ & $x \in[0,+\infty)$ \\
\hline & Gamma & $x^{k-1} \frac{e^{-x_{i} \theta}}{\theta^{k} \Gamma(k)}, k>0, \theta>0$ & $x \in[0,+\infty)$ \\
\hline $\mathrm{D}_{10}$ & Gumbel & $\frac{z \cdot \exp (-z)}{\beta}, z=\exp [-(x-\mu) / \beta], \mu \in R, \beta>0$ & $x \in(-\infty,+\infty)$ \\
\hline $\mathrm{D}_{11}$ & Hyperbolic Secant & $\frac{1}{2} \operatorname{sech}\left(\frac{\pi}{2} x\right)$ & $x \in(-\infty,+\infty)$ \\
\hline $\mathrm{D}_{12}$ & Laplace & $\frac{1}{2 b} \exp \left(-\frac{|x-\mu|}{b}\right), \mu \in R, b>0$ & $x \in(-\infty,+\infty)$ \\
\hline $\mathrm{D}_{13}$ & Logistic & $\frac{1}{4 s} \operatorname{sech}^{2}\left(\frac{x-\mu}{2 s}\right), \mu \in R_{s} s>0$ & $x \in(-\infty,+\infty)$ \\
\hline $\mathrm{D}_{14}$ & Lognormal & $\frac{1}{x \sigma \sqrt{2 \pi}} \exp \left[-\frac{(\operatorname{Inx} x-\mu)^{2}}{2 \sigma^{2}}\right], \mu \in R, \sigma^{2}>0$ & $x \in(0,+\infty)$ \\
\hline $\mathrm{D}_{15}$ & Normal & $\frac{1}{\sqrt{2 \pi \sigma^{2}}} \exp \left[-\frac{(\alpha-\mu)^{2}}{2 \sigma^{2}}\right], \mu \in R, \sigma^{2}>0$ & $x \in(0,+\infty)$ \\
\hline $\mathrm{D}_{16}$ & Pareto & $\frac{\alpha x_{m}^{\alpha}}{x^{\pi+1}}, x_{m}>0, \alpha>0$ & $x \in\left[x_{m},+\infty\right)$ \\
\hline $\mathrm{D}_{17}$ & Rayleigh & $\frac{x}{\sigma^{2}} \exp \left(-\frac{x^{2}}{2 \sigma^{2}}\right), \sigma>0$ & $x \in[0,+\infty)$ \\
\hline $\mathrm{D}_{18}$ & Semicircle & $\frac{2}{\pi r^{2}} \sqrt{r^{2}-x^{2}}, r>0$ & $x \in[-r, r]$ \\
\hline $\mathrm{D}_{19}$ & Student's-t & $\frac{\left(1+x^{2} / v\right)^{-\frac{w+1}{2}}}{\sqrt{v} E(1 / 2, v / 2)}, v>0$ & $x \in(-\infty,+\infty)$ \\
\hline $\mathrm{D}_{20}$ & Triangular & $\begin{cases}\frac{2(x-a)}{(b-a)(c-a)}, & a \leq x \leq c \\
\frac{2(b-x)}{(b-a)(b-c)}, & c \leq x \leq b\end{cases}$ & $x \in[a, b]$ \\
\hline $\mathrm{D}_{21}$ & Truncated Normal & $\frac{\frac{1}{\sigma} \phi\left(\frac{x-\mu}{\sigma}\right)}{\Phi\left(\frac{b-\mu}{\sigma}\right)-\Phi\left(\frac{(-\mu}{\sigma}\right)}, \mu \in R, a \in R, b \in R, \sigma^{2}>0$ & $x \in[a, b]$ \\
\hline $\mathrm{D}_{22}$ & Uniform & $\frac{1}{b-a}$ & $x \in[a, b]$ \\
\hline $\mathrm{D}_{23}$ & Von Mises & $\frac{e^{k \cos (x-\mu)}}{2 \pi I_{0}(k)}, \mu \in R, k>0$ & $x \in[-\pi, \pi]$ \\
\hline $\mathrm{D}_{24}$ & Weibull & $\frac{k}{\lambda}\left(\frac{x}{\lambda}\right)^{k-1} e^{-(x / \lambda)^{k}}, \lambda>0, k>0$ & $x \in[0,+\infty)$ \\
\hline
\end{tabular}


Note: $B(\alpha, \beta)=\int_{0}^{1} x^{\alpha-1}(1-x)^{\beta-1} d x$ is the Beta function. $\Psi(x)=d \ln [\Gamma(x)] / d x$ is the Digamma function, where $\Gamma(x)=\int_{0}^{\infty} u^{x-1} e^{-u} d u$ is the Gamma function. $I_{0}(x)$ is the modified Bessel function of order $0, I_{1}(x)$ and is the modified Bessel function of order 1 .

$$
Y_{M \times C}^{\prime}=H_{M \times L}^{\prime} \beta_{L \times C}=H_{M \times L}^{\prime} H_{L \times N}^{\dagger} Y_{N \times C}= \begin{cases}H^{\prime}\left(H^{T} H\right)^{-1} H^{T} Y, & \text { if } N \geq L \\ H^{\prime} H^{T}\left(H^{T}\right)^{-1} Y, & \text { if } N<L\end{cases}
$$

Table 2. The Details of 30 UCI Data Sets

\begin{tabular}{|c|c|c|c|c|c|}
\hline & Data sets & Attributes & Classes & Class distribution & Instances \\
\hline l & Auto Mpg & 5 & 3 & $245 / 79 / 68$ & 392 \\
\hline 2 & $\begin{array}{l}\text { Blood } \\
\text { Transfusion }\end{array}$ & 4 & 2 & $570 / 178$ & 748 \\
\hline 3 & Breast Cancer & 10 & 2 & $458 / 241$ & 699 \\
\hline 4 & $\begin{array}{l}\text { Breast Cancer } \\
\text { W-D }\end{array}$ & 30 & 2 & $357 / 212$ & 569 \\
\hline 5 & $\begin{array}{l}\text { Breast Cancer } \\
\text { W-P }\end{array}$ & 33 & 2 & $151 / 47$ & 198 \\
\hline 6 & Cleveland & 13 & 5 & $160 / 54 / 35 / 35 / 13$ & 297 \\
\hline 7 & Credit Approval & 15 & 2 & $383 / 307$ & 690 \\
\hline 8 & Cylinder Bands & 20 & 2 & $312 / 228$ & 540 \\
\hline 9 & Ecoli & 5 & 8 & $143 / 77 / 52 / 35 / 20 / 5 / 2 / 2$ & 336 \\
\hline 10 & $\begin{array}{l}\text { Glass } \\
\text { Identification }\end{array}$ & 9 & 7 & 76/70/29/17/13/9/0 & 214 \\
\hline 11 & $\begin{array}{l}\text { Haberman's } \\
\text { Survival }\end{array}$ & 3 & 2 & $225 / 81$ & 306 \\
\hline 12 & Heart Disease & 13 & 2 & $150 / 120$ & 270 \\
\hline 13 & Image Segment & 19 & 7 & $330 \times 7$ & 2310 \\
\hline 14 & Ionosphere & 33 & 2 & $225 / 126$ & 351 \\
\hline 15 & Iris & 4 & 3 & $50 \times 3$ & 150 \\
\hline 16 & Libras Movement & 90 & 15 & $24 \times 15$ & 360 \\
\hline 17 & Magic Telescope & 10 & 2 & $12332 / 6688$ & $19020(10 \%)$ \\
\hline 18 & Musk Version 1 & 166 & 2 & $269 / 207$ & 476 \\
\hline 19 & $\begin{array}{l}\text { New Thyroid } \\
\text { Gland }\end{array}$ & 5 & 3 & $150 / 35 / 30$ & 215 \\
\hline 20 & Page Blocks & 10 & 5 & $4913 / 329 / 115 / 88 / 28$ & $5473(10 \%)$ \\
\hline 21 & Parkinsons & 22 & 2 & $147 / 48$ & 195 \\
\hline 22 & $\begin{array}{l}\text { Pima Indian } \\
\text { Diabetes }\end{array}$ & 8 & 2 & $500 / 268$ & 768 \\
\hline 23 & Sonar & 60 & 2 & $111 / 97$ & 208 \\
\hline 24 & SPECTF Heart & 44 & 2 & $212 / 55$ & 267 \\
\hline 25 & $\begin{array}{l}\text { Vehicle } \\
\text { Silhouettes }\end{array}$ & 18 & 4 & $218 / 217 / 212 / 199$ & 846 \\
\hline 26 & $\begin{array}{l}\text { Vowel } \\
\text { Recognition }\end{array}$ & 10 & 11 & $48 \times 11$ & 528 \\
\hline 27 & Wine & 13 & 3 & $91 / 59 / 48$ & 178 \\
\hline 28 & Wine Quality-Red & 11 & 6 & $681 / 638 / 199 / 53 / 18 / 10$ & 1599 \\
\hline 29 & $\begin{array}{l}\text { Wine } \\
\text { Quality-White }\end{array}$ & 11 & 6 & $2198 / 1457 / 880 / 175 / 163 / 20$ & $4898(10 \%)$ \\
\hline 30 & Yeast & 8 & 10 & $463 / 429 / 244 / 163 / 51 / 44 / 35 / 30 / 20 / 5$ & 1484 \\
\hline
\end{tabular}

where $\beta_{L \times C}$ is the output-layer weights, $L$ is the number of hidden-layer nodes

$$
H_{N \times L}=\left[\begin{array}{c}
h\left(x_{1}\right) \\
h\left(x_{2}\right) \\
\vdots \\
h\left(x_{N}\right)
\end{array}\right]=\left[\begin{array}{cccc}
g\left(w_{1} x_{1}+b_{1}\right) & g\left(w_{2} x_{1}+b_{2}\right) & \cdots & g\left(w_{L} x_{1}+b_{L}\right) \\
g\left(w_{1} x_{2}+b_{1}\right) & g\left(w_{2} x_{2}+b_{2}\right) & \cdots & g\left(w_{L} x_{2}+b_{L}\right) \\
\vdots & \vdots & \ddots & \vdots \\
g\left(w_{L} x_{N}+b_{L}\right) & g\left(w_{2} x_{N}+b_{L}\right) & \cdots & g\left(w_{L} x_{N}+b_{L}\right)
\end{array}\right]
$$


is the hidden-layer output matrix for training instances, $\mathrm{H}_{L \times N}^{\dagger}$ is Moore-Penrose generalized inverse of $\mathrm{H}_{N \times L}$,

$$
H_{M \times L}^{\prime}=\left[\begin{array}{c}
h\left(x_{1}^{\prime}\right) \\
h\left(x_{2}^{\prime}\right) \\
\vdots \\
h\left(x_{M}^{\prime}\right)
\end{array}\right]=\left[\begin{array}{cccc}
g\left(w_{1} x_{1}^{\prime}+b_{1}\right) & g\left(w_{2} x_{1}^{\prime}+b_{2}\right) & \cdots & g\left(w_{L} x_{1}^{\prime}+b_{L}\right) \\
g\left(w_{1} x_{2}^{\prime}+b_{1}\right) & g\left(w_{2} x_{2}^{\prime}+b_{2}\right) & \cdots & g\left(w_{L} x_{2}^{\prime}+b_{L}\right) \\
\vdots & \vdots & \ddots & \vdots \\
g\left(w_{L} x_{M}^{\prime}+b_{L}\right) & g\left(w_{2} x_{M}^{\prime}+b_{L}\right) & \cdots & g\left(w_{L} x_{M}^{\prime}+b_{L}\right)
\end{array}\right]
$$

Table 3. Training Accuracies of ELMs Corresponding to 24 Probability Distributions on 30 UCI Data Sets

\begin{tabular}{|c|c|c|c|c|c|c|}
\hline & $\mathrm{D}_{1}$ :Beta & $\mathrm{D}_{2}$ :Cauchy & $\mathrm{D}_{3}$ :Central Chi & $\mathrm{D}_{4}:$ Chi & $\mathrm{D}_{5}$ :Erlang & $\mathrm{D}_{6}$ :Exponential \\
\hline 1 & $\begin{array}{c}0.858 \pm 0.002 \\
(1.5)\end{array}$ & $\begin{array}{c}0.848 \pm 0.003 \\
(1.5)\end{array}$ & $\begin{array}{c}0.863 \pm 0.003 \\
(7.5)\end{array}$ & $\begin{array}{c}0.867 \pm 0.002 \\
(24.0)\end{array}$ & $\begin{array}{c}0.867 \pm 0.003 \\
(5.0)\end{array}$ & $\begin{array}{c}0.862 \pm 0.002 \\
(5.0)\end{array}$ \\
\hline 2 & $\begin{array}{c}0.806 \pm 0.001 \\
(15.0)\end{array}$ & $\begin{array}{c}0.813 \pm 0.001 \\
(7.5)\end{array}$ & $\begin{array}{c}0.812 \pm 0.001 \\
\quad(13.0)\end{array}$ & $\begin{array}{c}0.810 \pm 0.001 \\
(2.0)\end{array}$ & $\begin{array}{c}0.812 \pm 0.001 \\
(21.5)\end{array}$ & $\begin{array}{c}0.811 \pm 0.001 \\
(21.5)\end{array}$ \\
\hline 3 & $\begin{array}{c}0.975 \pm 0.001 \\
(5.0)\end{array}$ & $\begin{array}{c}0.975 \pm 0.001 \\
(13.0)\end{array}$ & $\begin{array}{c}0.977 \pm 0.001 \\
(5.0)\end{array}$ & $\begin{array}{c}0.978 \pm 0.001 \\
(16.0)\end{array}$ & $\begin{array}{c}0.975 \pm 0.001 \\
(2.0)\end{array}$ & $\begin{array}{c}0.978 \pm 0.001 \\
(2.0)\end{array}$ \\
\hline 4 & $\begin{array}{c}0.976 \pm 0.002 \\
(17.0)\end{array}$ & $\begin{array}{c}0.954 \pm 0.004 \\
(23.0)\end{array}$ & $\begin{array}{c}0.849 \pm 0.004 \\
(14.5)\end{array}$ & $\begin{array}{c}0.905 \pm 0.003 \\
(24.0)\end{array}$ & $\begin{array}{c}0.842 \pm 0.003 \\
(13.0)\end{array}$ & $\begin{array}{c}0.935 \pm 0.003 \\
(14.5)\end{array}$ \\
\hline 5 & $\begin{array}{c}0.840 \pm 0.006 \\
(17.0)\end{array}$ & $\begin{array}{c}0.847 \pm 0.007 \\
(23.0)\end{array}$ & $\begin{array}{c}0.777 \pm 0.001 \\
\quad(13.5)\end{array}$ & $\begin{array}{c}0.799 \pm 0.002 \\
(24.0)\end{array}$ & $\begin{array}{c}0.773 \pm 0.002 \\
(13.5)\end{array}$ & $\begin{array}{c}0.818 \pm 0.003 \\
(15.0)\end{array}$ \\
\hline 6 & $\begin{array}{c}0.697 \pm 0.004 \\
(15.0)\end{array}$ & $\begin{array}{c}0.683 \pm 0.004 \\
(19.5)\end{array}$ & $\begin{array}{c}0.680 \pm 0.004 \\
(12.5)\end{array}$ & $\begin{array}{c}0.693 \pm 0.003 \\
(24.0)\end{array}$ & $\begin{array}{c}0.681 \pm 0.003 \\
(11.0)\end{array}$ & $\begin{array}{c}0.698 \pm 0.004 \\
(12.5)\end{array}$ \\
\hline 7 & $\begin{array}{c}0.804 \pm 0.002 \\
(10.0)\end{array}$ & $\begin{array}{c}0.809 \pm 0.003 \\
(2.5)\end{array}$ & $\begin{array}{c}0.812 \pm 0.002 \\
(9.0)\end{array}$ & $\begin{array}{c}0.807 \pm 0.002 \\
(2.5)\end{array}$ & $\begin{array}{c}0.812 \pm 0.002 \\
(13.0)\end{array}$ & $\begin{array}{c}0.808 \pm 0.002 \\
(13.0)\end{array}$ \\
\hline 8 & $\begin{array}{c}0.708 \pm 0.005 \\
(17.0)\end{array}$ & $\begin{array}{c}0.703 \pm 0.006 \\
\quad(24.0)\end{array}$ & $\begin{array}{c}0.599 \pm 0.003 \\
(15.5)\end{array}$ & $\begin{array}{c}0.647 \pm 0.005 \\
\quad(23.0)\end{array}$ & $\begin{array}{c}0.589 \pm 0.001 \\
(14.0)\end{array}$ & $\begin{array}{c}0.682 \pm 0.004 \\
(13.0)\end{array}$ \\
\hline 9 & $\begin{array}{c}0.904 \pm 0.002 \\
(17.0)\end{array}$ & $\begin{array}{c}0.899 \pm 0.002 \\
(20.5)\end{array}$ & $\begin{array}{c}0.895 \pm 0.002 \\
(12.5)\end{array}$ & $\begin{array}{c}0.897 \pm 0.002 \\
(24.0)\end{array}$ & $\begin{array}{c}0.894 \pm 0.002 \\
(12.5)\end{array}$ & $\begin{array}{c}0.900 \pm 0.001 \\
(14.5)\end{array}$ \\
\hline 10 & $\begin{array}{c}0.816 \pm 0.005 \\
(10.0)\end{array}$ & $\begin{array}{c}0.813 \pm 0.006 \\
(18.5)\end{array}$ & $\begin{array}{c}0.811 \pm 0.006 \\
(10.0)\end{array}$ & $\begin{array}{c}0.818 \pm 0.004 \\
(24.0)\end{array}$ & $\begin{array}{c}0.815 \pm 0.004 \\
(10.0)\end{array}$ & $\begin{array}{c}0.818 \pm 0.005 \\
(10.0)\end{array}$ \\
\hline 11 & $\begin{array}{c}0.784 \pm 0.002 \\
(18.0)\end{array}$ & $\begin{array}{c}0.800 \pm 0.003 \\
(10.5)\end{array}$ & $\begin{array}{c}0.798 \pm 0.003 \\
(16.0)\end{array}$ & $\begin{array}{c}0.791 \pm 0.002 \\
(17.0)\end{array}$ & $\begin{array}{c}0.799 \pm 0.003 \\
(20.5)\end{array}$ & $\begin{array}{c}0.795 \pm 0.003 \\
(22.5)\end{array}$ \\
\hline 12 & $\begin{array}{c}0.885 \pm 0.004 \\
(18.0)\end{array}$ & $\begin{array}{c}0.884 \pm 0.005 \\
\quad(22.5)\end{array}$ & $\begin{array}{c}0.812 \pm 0.004 \\
\quad(13.0)\end{array}$ & $\begin{array}{c}0.830 \pm 0.004 \\
\quad(24.0)\end{array}$ & $\begin{array}{c}0.800 \pm 0.005 \\
(15.0)\end{array}$ & $\begin{array}{c}0.864 \pm 0.005 \\
(14.0)\end{array}$ \\
\hline 13 & $\begin{array}{c}0.929 \pm 0.001 \\
(16.0)\end{array}$ & $\begin{array}{c}0.909 \pm 0.003 \\
(22.0)\end{array}$ & $\begin{array}{c}0.880 \pm 0.004 \\
(15.0)\end{array}$ & $\begin{array}{c}0.910 \pm 0.002 \\
(24.0)\end{array}$ & $\begin{array}{c}0.875 \pm 0.004 \\
(5.0)\end{array}$ & $\begin{array}{c}0.919 \pm 0.002 \\
(5.0)\end{array}$ \\
\hline 14 & $\begin{array}{c}0.861 \pm 0.003 \\
\quad(19.0)\end{array}$ & $\begin{array}{c}0.897 \pm 0.007 \\
(23.5)\end{array}$ & $\begin{array}{c}0.643 \pm 0.001 \\
\quad(13.0)\end{array}$ & $\begin{array}{c}0.652 \pm 0.002 \\
\quad(22.0)\end{array}$ & $\begin{array}{c}0.641 \pm 0.000 \\
(14.0)\end{array}$ & $\begin{array}{c}0.739 \pm 0.007 \\
(15.0)\end{array}$ \\
\hline 15 & $\begin{array}{c}0.988 \pm 0.001 \\
(20.0)\end{array}$ & $\begin{array}{c}0.984 \pm 0.002 \\
(5.0)\end{array}$ & $\begin{array}{c}0.986 \pm 0.001 \\
(20.0)\end{array}$ & $\begin{array}{c}0.985 \pm 0.001 \\
(5.0)\end{array}$ & $\begin{array}{c}0.987 \pm 0.001 \\
(20.0)\end{array}$ & $\begin{array}{c}0.985 \pm 0.001 \\
(20.0)\end{array}$ \\
\hline 16 & $\begin{array}{c}0.862 \pm 0.003 \\
(19.0)\end{array}$ & $\begin{array}{c}0.894 \pm 0.007 \\
(23.5)\end{array}$ & $\begin{array}{c}0.643 \pm 0.001 \\
(13.0)\end{array}$ & $\begin{array}{c}0.652 \pm 0.003 \\
\quad(22.0)\end{array}$ & $\begin{array}{c}0.641 \pm 0.000 \\
(14.0)\end{array}$ & $\begin{array}{c}0.736 \pm 0.007 \\
(15.0)\end{array}$ \\
\hline 17 & $\begin{array}{c}0.851 \pm 0.001 \\
(7.5)\end{array}$ & $\begin{array}{c}0.844 \pm 0.002 \\
\quad(21.0)\end{array}$ & $\begin{array}{c}0.843 \pm 0.002 \\
(6.0)\end{array}$ & $\begin{array}{c}0.848 \pm 0.002 \\
(24.0)\end{array}$ & $\begin{array}{c}0.840 \pm 0.002 \\
(3.0)\end{array}$ & $\begin{array}{c}0.850 \pm 0.001 \\
(3.0)\end{array}$ \\
\hline 18 & $\begin{array}{c}0.565 \pm 0.000 \\
(17.5)\end{array}$ & $\begin{array}{c}0.752 \pm 0.008 \\
(17.5)\end{array}$ & $\begin{array}{c}0.565 \pm 0.000 \\
(17.5)\end{array}$ & $\begin{array}{c}0.565 \pm 0.000 \\
(17.5)\end{array}$ & $\begin{array}{c}0.565 \pm 0.000 \\
(17.5)\end{array}$ & $\begin{array}{c}0.565 \pm 0.000 \\
(17.5)\end{array}$ \\
\hline 19 & $\begin{array}{c}0.952 \pm 0.001 \\
\quad(12.0)\end{array}$ & $\begin{array}{c}0.959 \pm 0.001 \\
(4.5)\end{array}$ & $\begin{array}{c}0.955 \pm 0.001 \\
(12.0)\end{array}$ & $\begin{array}{c}0.954 \pm 0.001 \\
(1.0)\end{array}$ & $\begin{array}{c}0.955 \pm 0.001 \\
(20.5)\end{array}$ & $\begin{array}{c}0.954 \pm 0.001 \\
(20.5)\end{array}$ \\
\hline 20 & $\begin{array}{c}0.970 \pm 0.001 \\
(3.0)\end{array}$ & $\begin{array}{c}0.973 \pm 0.002 \\
(14.5)\end{array}$ & $\begin{array}{c}0.972 \pm 0.001 \\
(1.0)\end{array}$ & $\begin{array}{c}0.974 \pm 0.001 \\
(20.5)\end{array}$ & $\begin{array}{c}0.971 \pm 0.001 \\
(3.0)\end{array}$ & $\begin{array}{c}0.975 \pm 0.001 \\
(6.5)\end{array}$ \\
\hline 21 & $\begin{array}{c}0.979 \pm 0.003 \\
(6.5)\end{array}$ & $\begin{array}{c}0.937 \pm 0.005 \\
\quad(21.0)\end{array}$ & $\begin{array}{c}0.941 \pm 0.005 \\
(6.5)\end{array}$ & $\begin{array}{c}0.975 \pm 0.003 \\
\quad(24.0)\end{array}$ & $\begin{array}{c}0.936 \pm 0.005 \\
(3.0)\end{array}$ & $\begin{array}{c}0.975 \pm 0.003 \\
(3.0)\end{array}$ \\
\hline 22 & $\begin{array}{c}0.803 \pm 0.001 \\
(17.0)\end{array}$ & $\begin{array}{c}0.796 \pm 0.002 \\
(21.5)\end{array}$ & $\begin{array}{c}0.787 \pm 0.003 \\
(15.0)\end{array}$ & $\begin{array}{c}0.795 \pm 0.001 \\
(24.0)\end{array}$ & $\begin{array}{c}0.784 \pm 0.002 \\
(13.0)\end{array}$ & $\begin{array}{c}0.798 \pm 0.002 \\
(13.0)\end{array}$ \\
\hline 23 & $\begin{array}{c}0.824 \pm 0.006 \\
(17.0)\end{array}$ & $\begin{array}{c}0.821 \pm 0.008 \\
\quad(22.5)\end{array}$ & $\begin{array}{l}0.534 \pm 0.000 \\
(13.0)\end{array}$ & $\begin{array}{l}0.541 \pm 0.003 \\
\quad(22.5)\end{array}$ & $\begin{array}{c}0.534 \pm 0.000 \\
\quad(14.0)\end{array}$ & $\begin{array}{c}0.642 \pm 0.007 \\
(15.0)\end{array}$ \\
\hline 24 & $\begin{array}{c}0.794 \pm 0.000 \\
(17.5)\end{array}$ & $\begin{array}{c}0.796 \pm 0.002 \\
(17.5)\end{array}$ & $\begin{array}{c}0.794 \pm 0.000 \\
(17.5)\end{array}$ & $\begin{array}{c}0.794 \pm 0.000 \\
(17.5)\end{array}$ & $\begin{array}{c}0.794 \pm 0.000 \\
(17.5)\end{array}$ & $\begin{array}{c}0.794 \pm 0.000 \\
(17.5)\end{array}$ \\
\hline 25 & $\begin{array}{c}0.791 \pm 0.003 \\
(16.0)\end{array}$ & $\begin{array}{c}0.746 \pm 0.006 \\
(21.0)\end{array}$ & $\begin{array}{c}0.726 \pm 0.006 \\
(12.5)\end{array}$ & $\begin{array}{c}0.762 \pm 0.004 \\
(24.0)\end{array}$ & $\begin{array}{c}0.723 \pm 0.004 \\
(4.0)\end{array}$ & $\begin{array}{c}0.771 \pm 0.004 \\
(4.0)\end{array}$ \\
\hline 26 & $\begin{array}{c}0.866 \pm 0.004 \\
(16.0)\end{array}$ & $\begin{array}{c}0.806 \pm 0.008 \\
\quad(21.0)\end{array}$ & $\begin{array}{l}0.761 \pm 0.008 \\
\quad(15.0)\end{array}$ & $\begin{array}{c}0.843 \pm 0.006 \\
\quad(24.0)\end{array}$ & $\begin{array}{c}0.754 \pm 0.009 \\
(14.0)\end{array}$ & $\begin{array}{c}0.846 \pm 0.005 \\
(12.5)\end{array}$ \\
\hline 27 & $0.999 \pm 0.001$ & $0.995 \pm 0.002$ & $0.939 \pm 0.004$ & $0.973 \pm 0.002$ & $0.926 \pm 0.005$ & $0.989 \pm 0.002$ \\
\hline
\end{tabular}




\begin{tabular}{|c|c|c|c|c|c|c|}
\hline & (17.0) & (21.0) & (13.0) & (24.0) & (14.5) & (14.5) \\
\hline 28 & $\begin{array}{c}0.628 \pm 0.002 \\
(9.0)\end{array}$ & $\begin{array}{c}0.623 \pm 0.003 \\
(20.0)\end{array}$ & $\begin{array}{c}0.619 \pm 0.002 \\
(9.0)\end{array}$ & $\begin{array}{c}0.628 \pm 0.002 \\
(24.0)\end{array}$ & $\begin{array}{c}0.621 \pm 0.002 \\
(2.5)\end{array}$ & $\begin{array}{c}0.628 \pm 0.002 \\
(2.5)\end{array}$ \\
\hline 29 & $0.635 \pm 0.003$ & $\begin{array}{c}0.634 \pm 0.005 \\
(7.5)\end{array}$ & $\begin{array}{c}0.639 \pm 0.005 \\
(3.5)\end{array}$ & $\begin{array}{c}0.643 \pm 0.004 \\
(24.0)\end{array}$ & $0.640 \pm 0.004$ & $0.643 \pm 0.004$ \\
\hline 30 & $\begin{array}{c}0.625 \pm 0.001 \\
(17.5)\end{array}$ & $\begin{array}{c}0.623 \pm 0.002 \\
(21.0)\end{array}$ & $\begin{array}{c}0.616 \pm 0.002 \\
(12.5)\end{array}$ & $\begin{array}{c}0.620 \pm 0.001 \\
(24.0)\end{array}$ & $\begin{array}{c}0.614 \pm 0.002 \\
(15.0)\end{array}$ & $\begin{array}{c}0.623 \pm 0.002 \\
(15.0)\end{array}$ \\
\hline \multirow[t]{2}{*}{ Rank } & $\begin{array}{ll}11.183 \\
\end{array}$ & $\begin{array}{l}13.700 \\
\end{array}$ & 1016.467 & $\begin{array}{l}13.717 \\
\end{array}$ & $\begin{array}{l}17.050 \\
\end{array}$ & 111.900 \\
\hline & $\mathrm{D}_{7}: \mathrm{F}$ & $\mathrm{D}_{8}:$ Frechet & $\mathrm{D}_{9}$ :Gamma & $\mathrm{D}_{10}$ :Gumbel & $\mathrm{D}_{11}$ :Hyperbolic & $\mathrm{D}_{12}$ :Laplace \\
\hline 1 & $\begin{array}{c}0.847 \pm 0.003 \\
(9.5)\end{array}$ & $\begin{array}{c}0.863 \pm 0.002 \\
(21.5)\end{array}$ & $\begin{array}{c}0.863 \pm 0.002 \\
(21.5)\end{array}$ & $\begin{array}{c}0.861 \pm 0.003 \\
(3.0)\end{array}$ & $\begin{array}{c}0.853 \pm 0.003 \\
(7.5)\end{array}$ & $\begin{array}{c}0.853 \pm 0.002 \\
(19.0)\end{array}$ \\
\hline 2 & $\begin{array}{c}0.813 \pm 0.001 \\
(2.0)\end{array}$ & $\begin{array}{c}0.806 \pm 0.001 \\
(13.0)\end{array}$ & $0.806 \pm 0.001$ & $0.813 \pm 0.001$ & $0.811 \pm 0.001$ & $\begin{array}{c}0.812 \pm 0.001 \\
(13.0)\end{array}$ \\
\hline 3 & $\begin{array}{c}0.974 \pm 0.001 \\
(10.5)\end{array}$ & $\begin{array}{c}0.979 \pm 0.001 \\
(20.5)\end{array}$ & $\begin{array}{c}0.979 \pm 0.001 \\
(16.0)\end{array}$ & $\begin{array}{c}0.976 \pm 0.001 \\
(10.5)\end{array}$ & $\begin{array}{c}0.973 \pm 0.001 \\
(5.0)\end{array}$ & $\begin{array}{c}0.974 \pm 0.001 \\
\quad(20.5)\end{array}$ \\
\hline 4 & $\begin{array}{c}0.720 \pm 0.039 \\
(21.0)\end{array}$ & $\begin{array}{c}0.936 \pm 0.002 \\
(3.0)\end{array}$ & $\begin{array}{c}0.935 \pm 0.002 \\
(3.0)\end{array}$ & $\begin{array}{c}0.848 \pm 0.005 \\
(22.0)\end{array}$ & $\begin{array}{c}0.977 \pm 0.001 \\
(18.0)\end{array}$ & $\begin{array}{c}0.977 \pm 0.001 \\
(3.0)\end{array}$ \\
\hline 5 & $\begin{array}{c}0.764 \pm 0.001 \\
(19.5)\end{array}$ & $\begin{array}{c}0.818 \pm 0.003 \\
(4.0)\end{array}$ & $\begin{array}{c}0.817 \pm 0.003 \\
(8.0)\end{array}$ & $\begin{array}{c}0.779 \pm 0.002 \\
(21.0)\end{array}$ & $\begin{array}{c}0.888 \pm 0.005 \\
(18.0)\end{array}$ & $\begin{array}{c}0.883 \pm 0.005 \\
(5.5)\end{array}$ \\
\hline 6 & $\begin{array}{c}0.653 \pm 0.004 \\
(23.0)\end{array}$ & $\begin{array}{c}0.699 \pm 0.004 \\
(5.5)\end{array}$ & $\begin{array}{c}0.698 \pm 0.004 \\
(9.5)\end{array}$ & $\begin{array}{c}0.675 \pm 0.004 \\
(22.0)\end{array}$ & $\begin{array}{c}0.703 \pm 0.004 \\
(17.0)\end{array}$ & $\begin{array}{c}0.701 \pm 0.004 \\
(5.5)\end{array}$ \\
\hline 7 & $\begin{array}{c}0.812 \pm 0.003 \\
(6.0)\end{array}$ & $\begin{array}{c}0.805 \pm 0.002 \\
(17.0)\end{array}$ & $\begin{array}{c}0.805 \pm 0.002 \\
(13.0)\end{array}$ & $\begin{array}{c}0.811 \pm 0.002 \\
(6.0)\end{array}$ & $\begin{array}{c}0.804 \pm 0.002 \\
(2.5)\end{array}$ & $\begin{array}{c}0.805 \pm 0.002 \\
(19.5)\end{array}$ \\
\hline 8 & $\begin{array}{c}0.590 \pm 0.003 \\
(19.0)\end{array}$ & $\begin{array}{c}0.688 \pm 0.005 \\
(2.0)\end{array}$ & $\begin{array}{c}0.689 \pm 0.004 \\
(6.5)\end{array}$ & $\begin{array}{c}0.601 \pm 0.003 \\
(22.0)\end{array}$ & $\begin{array}{c}0.722 \pm 0.005 \\
(18.0)\end{array}$ & $\begin{array}{c}0.719 \pm 0.006 \\
(4.5)\end{array}$ \\
\hline 9 & $\begin{array}{c}0.886 \pm 0.003 \\
(22.5)\end{array}$ & $0.900 \pm 0.002$ & $\begin{array}{c}0.899 \pm 0.001 \\
(9.0)\end{array}$ & $\begin{array}{c}0.893 \pm 0.002 \\
(22.5)\end{array}$ & $\begin{array}{c}0.905 \pm 0.002 \\
(17.0)\end{array}$ & $0.904 \pm 0.002$ \\
\hline 10 & $\begin{array}{c}0.785 \pm 0.005 \\
(23.0)\end{array}$ & $\begin{array}{c}0.818 \pm 0.005 \\
(2.5)\end{array}$ & $\begin{array}{c}0.818 \pm 0.004 \\
(2.5)\end{array}$ & $\begin{array}{c}0.804 \pm 0.004 \\
(20.0)\end{array}$ & $\begin{array}{c}0.820 \pm 0.006 \\
(14.5)\end{array}$ & $0.820 \pm 0.006$ \\
\hline 11 & $0.793 \pm 0.003$ & $\begin{array}{c}0.784 \pm 0.002 \\
(6.0)\end{array}$ & $\begin{array}{c}0.782 \pm 0.002 \\
(3.0)\end{array}$ & $\begin{array}{c}0.801 \pm 0.003 \\
(7.5)\end{array}$ & $\begin{array}{c}0.803 \pm 0.002 \\
(14.5)\end{array}$ & $\begin{array}{c}0.806 \pm 0.003 \\
(5.0)\end{array}$ \\
\hline 12 & $\begin{array}{c}0.795 \pm 0.006 \\
(21.0)\end{array}$ & $\begin{array}{c}0.856 \pm 0.003 \\
(2.0)\end{array}$ & $\begin{array}{c}0.857 \pm 0.004 \\
(4.0)\end{array}$ & $\begin{array}{c}0.809 \pm 0.006 \\
(22.5)\end{array}$ & $\begin{array}{c}0.889 \pm 0.004 \\
(17.0)\end{array}$ & $\begin{array}{c}0.888 \pm 0.004 \\
(6.5)\end{array}$ \\
\hline 13 & $\begin{array}{c}0.805 \pm 0.010 \\
(23.0)\end{array}$ & $\begin{array}{c}0.922 \pm 0.001 \\
(9.5)\end{array}$ & $\begin{array}{c}0.922 \pm 0.001 \\
(9.5)\end{array}$ & $\begin{array}{c}0.866 \pm 0.005 \\
(21.0)\end{array}$ & $\begin{array}{c}0.921 \pm 0.002 \\
(18.5)\end{array}$ & $\begin{array}{c}0.921 \pm 0.002 \\
(5.0)\end{array}$ \\
\hline 14 & $\begin{array}{c}0.642 \pm 0.001 \\
(18.0)\end{array}$ & $\begin{array}{c}0.701 \pm 0.004 \\
(1.0)\end{array}$ & $\begin{array}{c}0.693 \pm 0.004 \\
(8.0)\end{array}$ & $\begin{array}{c}0.653 \pm 0.003 \\
(20.0)\end{array}$ & $\begin{array}{c}0.936 \pm 0.003 \\
(17.0)\end{array}$ & $\begin{array}{c}0.931 \pm 0.004 \\
(4.5)\end{array}$ \\
\hline 15 & $\begin{array}{c}0.987 \pm 0.002 \\
(20.0)\end{array}$ & $\begin{array}{c}0.985 \pm 0.001 \\
(12.0)\end{array}$ & $\begin{array}{c}0.985 \pm 0.001 \\
(12.0)\end{array}$ & $\begin{array}{c}0.985 \pm 0.001 \\
(20.0)\end{array}$ & $\begin{array}{c}0.986 \pm 0.001 \\
(20.0)\end{array}$ & $\begin{array}{c}0.986 \pm 0.001 \\
(12.0)\end{array}$ \\
\hline 16 & $\begin{array}{c}0.642 \pm 0.001 \\
(18.0)\end{array}$ & $\begin{array}{c}0.703 \pm 0.005 \\
(1.0)\end{array}$ & $\begin{array}{c}0.693 \pm 0.004 \\
(8.5)\end{array}$ & $\begin{array}{c}0.654 \pm 0.003 \\
(20.0)\end{array}$ & $\begin{array}{c}0.936 \pm 0.003 \\
(17.0)\end{array}$ & $0.930 \pm 0.004$ \\
\hline 17 & $\begin{array}{c}0.830 \pm 0.004 \\
(22.0)\end{array}$ & $\begin{array}{c}0.851 \pm 0.001 \\
(11.5)\end{array}$ & $\begin{array}{c}0.851 \pm 0.001 \\
(11.5)\end{array}$ & $\begin{array}{c}0.838 \pm 0.002 \\
(23.0)\end{array}$ & $\begin{array}{c}0.847 \pm 0.001 \\
(7.5)\end{array}$ & $\begin{array}{c}0.847 \pm 0.001 \\
(16.5)\end{array}$ \\
\hline 18 & $\begin{array}{c}0.565 \pm 0.000 \\
(17.5)\end{array}$ & $\begin{array}{c}0.565 \pm 0.000 \\
(4.0)\end{array}$ & $\begin{array}{c}0.565 \pm 0.000 \\
(9.0)\end{array}$ & $\begin{array}{c}0.565 \pm 0.000 \\
(17.5)\end{array}$ & $\begin{array}{c}0.809 \pm 0.007 \\
(17.5)\end{array}$ & $\begin{array}{c}0.798 \pm 0.008 \\
(6.0)\end{array}$ \\
\hline 19 & $\begin{array}{c}0.960 \pm 0.001 \\
(12.0)\end{array}$ & $\begin{array}{c}0.953 \pm 0.001 \\
(12.0)\end{array}$ & $\begin{array}{c}0.953 \pm 0.001 \\
(12.0)\end{array}$ & $\begin{array}{c}0.954 \pm 0.001 \\
(12.0)\end{array}$ & $\begin{array}{c}0.954 \pm 0.001 \\
(3.0)\end{array}$ & $\begin{array}{c}0.954 \pm 0.001 \\
(12.0)\end{array}$ \\
\hline 20 & $\begin{array}{c}0.970 \pm 0.001 \\
(10.5)\end{array}$ & $\begin{array}{c}0.974 \pm 0.001 \\
(20.5)\end{array}$ & $\begin{array}{c}0.973 \pm 0.001 \\
(14.5)\end{array}$ & $\begin{array}{c}0.972 \pm 0.001 \\
(10.5)\end{array}$ & $\begin{array}{c}0.970 \pm 0.001 \\
(3.0)\end{array}$ & $\begin{array}{c}0.971 \pm 0.001 \\
(20.5)\end{array}$ \\
\hline 21 & $\begin{array}{c}0.879 \pm 0.007 \\
(23.0)\end{array}$ & $\begin{array}{c}0.981 \pm 0.003 \\
\quad(12.0)\end{array}$ & $\begin{array}{c}0.981 \pm 0.003 \\
(10.0)\end{array}$ & $\begin{array}{c}0.929 \pm 0.005 \\
(22.0)\end{array}$ & $\begin{array}{c}0.946 \pm 0.004 \\
(9.0)\end{array}$ & $\begin{array}{c}0.947 \pm 0.006 \\
(14.5)\end{array}$ \\
\hline 22 & $\begin{array}{c}0.777 \pm 0.003 \\
(23.0)\end{array}$ & $\begin{array}{c}0.799 \pm 0.001 \\
(7.5)\end{array}$ & $\begin{array}{c}0.799 \pm 0.001 \\
(11.0)\end{array}$ & $\begin{array}{c}0.783 \pm 0.003 \\
(21.5)\end{array}$ & $\begin{array}{c}0.802 \pm 0.002 \\
(18.0)\end{array}$ & $\begin{array}{c}0.801 \pm 0.002 \\
(7.5)\end{array}$ \\
\hline 23 & $\begin{array}{c}0.534 \pm 0.000 \\
(19.0)\end{array}$ & $\begin{array}{c}0.593 \pm 0.008 \\
(6.0)\end{array}$ & $\begin{array}{c}0.573 \pm 0.005 \\
(8.0)\end{array}$ & $\begin{array}{c}0.537 \pm 0.003 \\
(20.0)\end{array}$ & $\begin{array}{c}0.891 \pm 0.008 \\
(18.0)\end{array}$ & $\begin{array}{c}0.881 \pm 0.007 \\
(4.5)\end{array}$ \\
\hline 24 & $\begin{array}{c}0.794 \pm 0.000 \\
(17.5)\end{array}$ & $\begin{array}{c}0.794 \pm 0.000 \\
(5.5)\end{array}$ & $\begin{array}{c}0.794 \pm 0.000 \\
(8.0)\end{array}$ & $\begin{array}{c}0.794 \pm 0.000 \\
(17.5)\end{array}$ & $\begin{array}{c}0.824 \pm 0.005 \\
(17.5)\end{array}$ & $\begin{array}{c}0.818 \pm 0.004 \\
(4.0)\end{array}$ \\
\hline 25 & $\begin{array}{c}0.669 \pm 0.008 \\
(23.0)\end{array}$ & $\begin{array}{c}0.781 \pm 0.003 \\
(6.0)\end{array}$ & $\begin{array}{c}0.781 \pm 0.003 \\
(9.5)\end{array}$ & $\begin{array}{c}0.710 \pm 0.005 \\
(22.0)\end{array}$ & $\begin{array}{c}0.774 \pm 0.004 \\
(18.0)\end{array}$ & $\begin{array}{c}0.772 \pm 0.004 \\
(7.5)\end{array}$ \\
\hline 26 & $\begin{array}{c}0.599 \pm 0.015 \\
(23.0)\end{array}$ & $\begin{array}{c}0.857 \pm 0.004 \\
(1.5)\end{array}$ & $\begin{array}{c}0.858 \pm 0.004 \\
(3.5)\end{array}$ & $\begin{array}{c}0.710 \pm 0.009 \\
(22.0)\end{array}$ & $\begin{array}{c}0.868 \pm 0.005 \\
(18.0)\end{array}$ & $\begin{array}{c}0.867 \pm 0.004 \\
(5.5)\end{array}$ \\
\hline 27 & $\begin{array}{c}0.874 \pm 0.012 \\
(22.0)\end{array}$ & $\begin{array}{c}0.988 \pm 0.001 \\
(5.0)\end{array}$ & $\begin{array}{c}0.988 \pm 0.001 \\
(5.0)\end{array}$ & $\begin{array}{c}0.921 \pm 0.007 \\
(23.0)\end{array}$ & $\begin{array}{c}1.000 \pm 0.000 \\
(18.0)\end{array}$ & $\begin{array}{c}1.000 \pm 0.000 \\
(5.0)\end{array}$ \\
\hline 28 & $0.601 \pm 0.003$ & $0.629 \pm 0.002$ & $0.629 \pm 0.002$ & $0.615 \pm 0.003$ & $0.628 \pm 0.002$ & $0.628 \pm 0.002$ \\
\hline
\end{tabular}




\begin{tabular}{|c|c|c|c|c|c|c|}
\hline & (23.0) & $(9.0)$ & $(9.0)$ & (22.0) & (18.5) & (9.0) \\
\hline 29 & $\begin{array}{c}0.604 \pm 0.006 \\
\quad(23.0)\end{array}$ & $\begin{array}{c}0.640 \pm 0.005 \\
(17.5)\end{array}$ & $\begin{array}{c}0.642 \pm 0.004 \\
(11.5)\end{array}$ & $\begin{array}{c}0.631 \pm 0.005 \\
(20.0)\end{array}$ & $\begin{array}{c}0.636 \pm 0.005 \\
(2.0)\end{array}$ & $\begin{array}{c}0.638 \pm 0.005 \\
(15.0)\end{array}$ \\
\hline 30 & $\begin{array}{c}0.601 \pm 0.003 \\
(22.5) \\
\end{array}$ & $\begin{array}{c}0.622 \pm 0.001 \\
(4.5)\end{array}$ & $\begin{array}{c}0.622 \pm 0.001 \\
(1.0)\end{array}$ & $\begin{array}{c}0.613 \pm 0.002 \\
(22.5) \\
\end{array}$ & $\begin{array}{c}0.625 \pm 0.002 \\
(17.5) \\
\end{array}$ & $\begin{array}{c}0.626 \pm 0.001 \\
(4.5) \\
\end{array}$ \\
\hline \multirow[t]{2}{*}{ Rank } & 19.883 & 11.767 & 12.067 & 18.150 & 8.267 & 8.833 \\
\hline & $\mathrm{D}_{13}$ :Logistic & $\mathrm{D}_{14}$ :Lognormal & $\mathrm{D}_{15}$ : Normal & $\mathrm{D}_{16}$ :Pareto & $\mathrm{D}_{17}:$ Rayleigh & $\mathrm{D}_{18}:$ Semicircle \\
\hline 1 & $\begin{array}{c}0.865 \pm 0.003 \\
(13.0)\end{array}$ & $\begin{array}{c}0.862 \pm 0.003 \\
(23.0)\end{array}$ & $\begin{array}{c}0.854 \pm 0.003 \\
(5.0)\end{array}$ & $\begin{array}{c}0.859 \pm 0.003 \\
(11.5)\end{array}$ & $\begin{array}{c}0.861 \pm 0.003 \\
(9.5)\end{array}$ & $\begin{array}{c}0.855 \pm 0.002 \\
(16.0)\end{array}$ \\
\hline 2 & $\begin{array}{c}0.812 \pm 0.001 \\
(21.5)\end{array}$ & $\begin{array}{c}0.812 \pm 0.001 \\
(2.0)\end{array}$ & $\begin{array}{c}0.811 \pm 0.001 \\
(7.5)\end{array}$ & $\begin{array}{c}0.807 \pm 0.001 \\
(17.5)\end{array}$ & $\begin{array}{c}0.807 \pm 0.001 \\
(17.5)\end{array}$ & $\begin{array}{c}0.806 \pm 0.001 \\
(21.5)\end{array}$ \\
\hline 3 & $\begin{array}{c}0.976 \pm 0.001 \\
(13.0)\end{array}$ & $\begin{array}{c}0.978 \pm 0.001 \\
(13.0)\end{array}$ & $\begin{array}{c}0.973 \pm 0.001 \\
(8.0)\end{array}$ & $\begin{array}{c}0.977 \pm 0.001 \\
(8.0)\end{array}$ & $\begin{array}{c}0.977 \pm 0.001 \\
(8.0)\end{array}$ & $\begin{array}{c}0.973 \pm 0.001 \\
(20.5)\end{array}$ \\
\hline 4 & $\begin{array}{c}0.846 \pm 0.004 \\
(7.0)\end{array}$ & $\begin{array}{c}0.885 \pm 0.005 \\
(12.0)\end{array}$ & $\begin{array}{c}0.977 \pm 0.001 \\
(20.0)\end{array}$ & $\begin{array}{c}0.882 \pm 0.003 \\
(19.0)\end{array}$ & $\begin{array}{c}0.965 \pm 0.002 \\
(11.0)\end{array}$ & $\begin{array}{c}0.975 \pm 0.001 \\
(9.5)\end{array}$ \\
\hline 5 & $\begin{array}{c}0.778 \pm 0.002 \\
(11.0)\end{array}$ & $\begin{array}{c}0.792 \pm 0.002 \\
(10.0)\end{array}$ & $\begin{array}{c}0.886 \pm 0.006 \\
(22.0)\end{array}$ & $\begin{array}{c}0.779 \pm 0.001 \\
(19.5)\end{array}$ & $\begin{array}{c}0.825 \pm 0.005 \\
(12.0)\end{array}$ & $\begin{array}{c}0.892 \pm 0.004 \\
(1.5)\end{array}$ \\
\hline 6 & $\begin{array}{c}0.679 \pm 0.004 \\
(14.0)\end{array}$ & $\begin{array}{c}0.685 \pm 0.004 \\
(18.0)\end{array}$ & $\begin{array}{c}0.703 \pm 0.003 \\
\quad(21.0)\end{array}$ & $\begin{array}{c}0.681 \pm 0.004 \\
(19.5)\end{array}$ & $\begin{array}{c}0.702 \pm 0.003 \\
(7.5)\end{array}$ & $\begin{array}{c}0.706 \pm 0.004 \\
(1.5)\end{array}$ \\
\hline 7 & $\begin{array}{c}0.811 \pm 0.002 \\
(17.0)\end{array}$ & $\begin{array}{c}0.812 \pm 0.002 \\
(8.0)\end{array}$ & $\begin{array}{c}0.803 \pm 0.003 \\
(2.5)\end{array}$ & $\begin{array}{c}0.811 \pm 0.002 \\
(6.0)\end{array}$ & $\begin{array}{c}0.805 \pm 0.002 \\
(13.0)\end{array}$ & $\begin{array}{c}0.802 \pm 0.002 \\
(22.0)\end{array}$ \\
\hline 8 & $\begin{array}{c}0.597 \pm 0.002 \\
(10.0)\end{array}$ & $\begin{array}{c}0.629 \pm 0.005 \\
(11.0)\end{array}$ & $\begin{array}{c}0.721 \pm 0.005 \\
(20.5)\end{array}$ & $\begin{array}{c}0.599 \pm 0.002 \\
(20.5)\end{array}$ & $\begin{array}{c}0.702 \pm 0.005 \\
\quad(12.0)\end{array}$ & $\begin{array}{c}0.721 \pm 0.006 \\
(4.5)\end{array}$ \\
\hline 9 & $\begin{array}{c}0.893 \pm 0.002 \\
(9.0)\end{array}$ & $\begin{array}{c}0.897 \pm 0.002 \\
(14.5)\end{array}$ & $\begin{array}{c}0.905 \pm 0.002 \\
(19.0)\end{array}$ & $\begin{array}{c}0.894 \pm 0.001 \\
(20.5)\end{array}$ & $\begin{array}{c}0.904 \pm 0.002 \\
(9.0)\end{array}$ & $\begin{array}{c}0.906 \pm 0.001 \\
(2.0)\end{array}$ \\
\hline 10 & $\begin{array}{c}0.814 \pm 0.005 \\
(16.5)\end{array}$ & $\begin{array}{c}0.817 \pm 0.005 \\
(21.0)\end{array}$ & $\begin{array}{c}0.819 \pm 0.006 \\
(22.0)\end{array}$ & $\begin{array}{c}0.820 \pm 0.004 \\
(2.5)\end{array}$ & $\begin{array}{c}0.817 \pm 0.005 \\
(14.5)\end{array}$ & $\begin{array}{c}0.816 \pm 0.005 \\
(16.5)\end{array}$ \\
\hline 11 & $\begin{array}{c}0.801 \pm 0.003 \\
\quad(20.5)\end{array}$ & $\begin{array}{c}0.796 \pm 0.002 \\
(9.0)\end{array}$ & $\begin{array}{c}0.805 \pm 0.003 \\
(12.5)\end{array}$ & $\begin{array}{c}0.781 \pm 0.003 \\
(24.0)\end{array}$ & $\begin{array}{c}0.787 \pm 0.002 \\
(19.0)\end{array}$ & $\begin{array}{c}0.798 \pm 0.003 \\
(12.5)\end{array}$ \\
\hline 12 & $\begin{array}{c}0.800 \pm 0.006 \\
(9.5)\end{array}$ & $\begin{array}{c}0.832 \pm 0.005 \\
(11.0)\end{array}$ & $\begin{array}{c}0.887 \pm 0.004 \\
(20.0)\end{array}$ & $\begin{array}{c}0.817 \pm 0.004 \\
(19.0)\end{array}$ & $\begin{array}{c}0.879 \pm 0.004 \\
(12.0)\end{array}$ & $\begin{array}{c}0.887 \pm 0.004 \\
(6.5)\end{array}$ \\
\hline 13 & $\begin{array}{c}0.876 \pm 0.005 \\
(1.0)\end{array}$ & $\begin{array}{c}0.901 \pm 0.003 \\
(17.0)\end{array}$ & $\begin{array}{c}0.922 \pm 0.002 \\
\quad(20.0)\end{array}$ & $\begin{array}{c}0.901 \pm 0.002 \\
(18.5)\end{array}$ & $\begin{array}{c}0.927 \pm 0.001 \\
(2.0)\end{array}$ & $\begin{array}{c}0.920 \pm 0.001 \\
(13.0)\end{array}$ \\
\hline 14 & $\begin{array}{c}0.648 \pm 0.001 \\
(11.0)\end{array}$ & $\begin{array}{c}0.659 \pm 0.003 \\
(10.0)\end{array}$ & $\begin{array}{c}0.934 \pm 0.004 \\
(21.0)\end{array}$ & $\begin{array}{c}0.641 \pm 0.000 \\
(23.5)\end{array}$ & $\begin{array}{c}0.846 \pm 0.003 \\
(12.0)\end{array}$ & $\begin{array}{c}0.934 \pm 0.003 \\
(4.5)\end{array}$ \\
\hline 15 & $\begin{array}{c}0.985 \pm 0.002 \\
(1.5)\end{array}$ & $\begin{array}{c}0.985 \pm 0.002 \\
(24.0)\end{array}$ & $\begin{array}{c}0.986 \pm 0.002 \\
(12.0)\end{array}$ & $\begin{array}{c}0.988 \pm 0.001 \\
(1.5)\end{array}$ & $\begin{array}{c}0.986 \pm 0.001 \\
(12.0)\end{array}$ & $\begin{array}{c}0.987 \pm 0.001 \\
(5.0)\end{array}$ \\
\hline 16 & $\begin{array}{c}0.648 \pm 0.002 \\
(11.0)\end{array}$ & $\begin{array}{c}0.659 \pm 0.003 \\
(10.0)\end{array}$ & $\begin{array}{c}0.935 \pm 0.004 \\
\quad(21.0)\end{array}$ & $\begin{array}{c}0.641 \pm 0.000 \\
(23.5)\end{array}$ & $\begin{array}{c}0.846 \pm 0.003 \\
(12.0)\end{array}$ & $\begin{array}{c}0.933 \pm 0.003 \\
(6.0)\end{array}$ \\
\hline 17 & $\begin{array}{c}0.836 \pm 0.002 \\
(3.0)\end{array}$ & $\begin{array}{c}0.848 \pm 0.002 \\
(19.0)\end{array}$ & $\begin{array}{c}0.846 \pm 0.001 \\
(20.0)\end{array}$ & $\begin{array}{c}0.847 \pm 0.002 \\
(11.5)\end{array}$ & $\begin{array}{c}0.851 \pm 0.001 \\
(3.0)\end{array}$ & $\begin{array}{c}0.846 \pm 0.001 \\
(16.5)\end{array}$ \\
\hline 18 & $\begin{array}{c}0.565 \pm 0.000 \\
(17.5)\end{array}$ & $\begin{array}{c}0.565 \pm 0.000 \\
(10.0)\end{array}$ & $\begin{array}{c}0.806 \pm 0.007 \\
(17.5)\end{array}$ & $\begin{array}{c}0.565 \pm 0.000 \\
(17.5)\end{array}$ & $\begin{array}{c}0.565 \pm 0.000 \\
(17.5)\end{array}$ & $\begin{array}{c}0.823 \pm 0.007 \\
(2.0)\end{array}$ \\
\hline 19 & $\begin{array}{c}0.954 \pm 0.001 \\
(23.0)\end{array}$ & $\begin{array}{c}0.956 \pm 0.001 \\
(2.0)\end{array}$ & $\begin{array}{c}0.954 \pm 0.001 \\
(4.5)\end{array}$ & $\begin{array}{c}0.954 \pm 0.001 \\
(12.0)\end{array}$ & $\begin{array}{c}0.953 \pm 0.001 \\
(20.5)\end{array}$ & $\begin{array}{c}0.954 \pm 0.001 \\
(12.0)\end{array}$ \\
\hline 20 & $\begin{array}{c}0.972 \pm 0.001 \\
\quad(20.5)\end{array}$ & $0.974 \pm 0.001$ & $\begin{array}{c}0.970 \pm 0.001 \\
(10.5)\end{array}$ & $\begin{array}{c}0.973 \pm 0.001 \\
(6.5)\end{array}$ & $\begin{array}{c}0.972 \pm 0.001 \\
\quad(10.5)\end{array}$ & $\begin{array}{c}0.970 \pm 0.001 \\
\quad(20.5)\end{array}$ \\
\hline 21 & $\begin{array}{c}0.933 \pm 0.005 \\
(5.0)\end{array}$ & $\begin{array}{c}0.962 \pm 0.004 \\
(20.0)\end{array}$ & $\begin{array}{c}0.945 \pm 0.005 \\
(17.5)\end{array}$ & $\begin{array}{c}0.973 \pm 0.003 \\
(8.0)\end{array}$ & $\begin{array}{c}0.981 \pm 0.002 \\
(3.0)\end{array}$ & $\begin{array}{c}0.941 \pm 0.003 \\
(17.5)\end{array}$ \\
\hline 22 & $\begin{array}{c}0.784 \pm 0.002 \\
(2.5)\end{array}$ & $\begin{array}{c}0.793 \pm 0.002 \\
(16.0)\end{array}$ & $\begin{array}{c}0.802 \pm 0.002 \\
(20.0)\end{array}$ & $\begin{array}{c}0.792 \pm 0.002 \\
(19.0)\end{array}$ & $\begin{array}{c}0.802 \pm 0.002 \\
(7.5)\end{array}$ & $\begin{array}{c}0.803 \pm 0.002 \\
(2.5)\end{array}$ \\
\hline 23 & $\begin{array}{c}0.535 \pm 0.001 \\
(10.0)\end{array}$ & $\begin{array}{c}0.540 \pm 0.003 \\
(11.0)\end{array}$ & $\begin{array}{c}0.892 \pm 0.007 \\
\quad(22.5)\end{array}$ & $\begin{array}{c}0.534 \pm 0.000 \\
(22.5)\end{array}$ & $\begin{array}{c}0.765 \pm 0.005 \\
(12.0)\end{array}$ & $\begin{array}{c}0.895 \pm 0.007 \\
(3.0)\end{array}$ \\
\hline 24 & $\begin{array}{c}0.794 \pm 0.000 \\
(17.5)\end{array}$ & $\begin{array}{c}0.794 \pm 0.000 \\
(10.0)\end{array}$ & $\begin{array}{c}0.825 \pm 0.004 \\
(17.5)\end{array}$ & $\begin{array}{c}0.794 \pm 0.000 \\
(17.5)\end{array}$ & $\begin{array}{c}0.794 \pm 0.000 \\
(17.5)\end{array}$ & $\begin{array}{c}0.842 \pm 0.005 \\
(1.0)\end{array}$ \\
\hline 25 & $\begin{array}{c}0.712 \pm 0.006 \\
(1.0)\end{array}$ & $\begin{array}{c}0.748 \pm 0.004 \\
\quad(19.0)\end{array}$ & $\begin{array}{c}0.773 \pm 0.004 \\
\quad(20.0)\end{array}$ & $\begin{array}{c}0.754 \pm 0.004 \\
(17.0)\end{array}$ & $\begin{array}{c}0.786 \pm 0.004 \\
(2.0)\end{array}$ & $\begin{array}{c}0.771 \pm 0.004 \\
\quad(12.5)\end{array}$ \\
\hline 26 & $\begin{array}{c}0.739 \pm 0.009 \\
(5.5)\end{array}$ & $\begin{array}{c}0.811 \pm 0.007 \\
(19.0)\end{array}$ & $\begin{array}{c}0.866 \pm 0.004 \\
(20.0)\end{array}$ & $\begin{array}{c}0.836 \pm 0.006 \\
(17.0)\end{array}$ & $\begin{array}{c}0.862 \pm 0.005 \\
\quad(11.0)\end{array}$ & $\begin{array}{c}0.865 \pm 0.005 \\
(7.5)\end{array}$ \\
\hline 27 & $\begin{array}{c}0.920 \pm 0.006 \\
(10.0)\end{array}$ & $\begin{array}{c}0.967 \pm 0.003 \\
(12.0)\end{array}$ & $\begin{array}{c}1.000 \pm 0.000 \\
(20.0)\end{array}$ & $\begin{array}{c}0.966 \pm 0.002 \\
(19.0)\end{array}$ & $\begin{array}{l}0.998 \pm 0.001 \\
\quad(11.0)\end{array}$ & $\begin{array}{c}1.000 \pm 0.000 \\
(5.0)\end{array}$ \\
\hline 28 & $\begin{array}{c}0.617 \pm 0.002 \\
(9.0)\end{array}$ & $\begin{array}{c}0.623 \pm 0.002 \\
(18.5)\end{array}$ & $\begin{array}{c}0.628 \pm 0.002 \\
(21.0)\end{array}$ & $\begin{array}{c}0.626 \pm 0.002 \\
(16.5)\end{array}$ & $\begin{array}{c}0.628 \pm 0.002 \\
(9.0)\end{array}$ & $\begin{array}{c}0.626 \pm 0.002 \\
(16.5)\end{array}$ \\
\hline 29 & $0.635 \pm 0.005$ & $0.645 \pm 0.004$ & $0.637 \pm 0.004$ & $0.646 \pm 0.004$ & $0.638 \pm 0.004$ & $0.636 \pm 0.004$ \\
\hline
\end{tabular}




\begin{tabular}{|c|c|c|c|c|c|c|}
\hline 30 & $\begin{array}{c}(20.0) \\
0.613 \pm 0.002 \\
(4.5) \\
\end{array}$ & $\begin{array}{c}(22.0) \\
0.620 \pm 0.001 \\
(12.5) \\
\end{array}$ & $\begin{array}{c}(9.0) \\
0.625 \pm 0.001 \\
(20.0) \\
\end{array}$ & $\begin{array}{c}(1.0) \\
0.619 \pm 0.001 \\
(19.0) \\
\end{array}$ & $\begin{array}{c}(11.5) \\
0.624 \pm 0.001 \\
(9.5) \\
\end{array}$ & $\begin{array}{c}(17.5) \\
0.624 \pm 0.002 \\
(9.5) \\
\end{array}$ \\
\hline \multirow[t]{2}{*}{ Rank } & $\begin{array}{l}18.100 \\
\end{array}$ & $\begin{array}{l}13.817 \\
\end{array}$ & 8.983 & 15.283 & 10.950 & $\begin{array}{ll}10.217 \\
\end{array}$ \\
\hline & $\mathrm{D}_{19}:$ Student $\mathrm{i}_{\mathrm{i}} \mathrm{s}-\mathrm{t}$ & $\mathrm{D}_{20}$ :Triangular & $\mathrm{D}_{21}$ :Truncated & $\mathrm{D}_{22}$ :Uniform & $\mathrm{D}_{23}$ :Von Mises & $\mathrm{D}_{24}$ :Weibull \\
\hline 1 & $\begin{array}{c}0.854 \pm 0.002 \\
(19.0)\end{array}$ & $\begin{array}{c}0.855 \pm 0.003 \\
(16.0)\end{array}$ & $\begin{array}{c}0.856 \pm 0.002 \\
(14.0)\end{array}$ & $\begin{array}{c}0.855 \pm 0.002 \\
(16.0)\end{array}$ & $\begin{array}{c}0.854 \pm 0.002 \\
(19.0)\end{array}$ & $\begin{array}{c}0.859 \pm 0.002 \\
(11.5)\end{array}$ \\
\hline 2 & $\begin{array}{c}0.812 \pm 0.001 \\
(7.5)\end{array}$ & $\begin{array}{c}0.812 \pm 0.001 \\
(7.5)\end{array}$ & $\begin{array}{c}0.807 \pm 0.001 \\
(17.5)\end{array}$ & $\begin{array}{c}0.812 \pm 0.001 \\
(7.5)\end{array}$ & $\begin{array}{c}0.807 \pm 0.001 \\
\quad(17.5)\end{array}$ & $\begin{array}{c}0.805 \pm 0.001 \\
(24.0)\end{array}$ \\
\hline 3 & $\begin{array}{c}0.973 \pm 0.001 \\
(20.5)\end{array}$ & $\begin{array}{c}0.973 \pm 0.001 \\
(20.5)\end{array}$ & $\begin{array}{c}0.973 \pm 0.001 \\
(20.5)\end{array}$ & $\begin{array}{c}0.974 \pm 0.001 \\
(16.0)\end{array}$ & $\begin{array}{l}0.972 \pm 0.001 \\
\quad(24.0)\end{array}$ & $\begin{array}{c}0.979 \pm 0.001 \\
(2.0)\end{array}$ \\
\hline 4 & $\begin{array}{c}0.977 \pm 0.001 \\
(3.0)\end{array}$ & $\begin{array}{c}0.977 \pm 0.001 \\
(3.0)\end{array}$ & $\begin{array}{c}0.975 \pm 0.001 \\
(9.5)\end{array}$ & $\begin{array}{c}0.976 \pm 0.001 \\
(7.0)\end{array}$ & $\begin{array}{c}0.976 \pm 0.001 \\
(7.0)\end{array}$ & $\begin{array}{c}0.933 \pm 0.002 \\
(16.0)\end{array}$ \\
\hline 5 & $\begin{array}{c}0.885 \pm 0.005 \\
(7.0)\end{array}$ & $0.886 \pm 0.006$ & $\begin{array}{c}0.892 \pm 0.005 \\
(1.5)\end{array}$ & $\begin{array}{c}0.881 \pm 0.006 \\
(9.0)\end{array}$ & $\begin{array}{c}0.891 \pm 0.004 \\
(3.0)\end{array}$ & $\begin{array}{c}0.811 \pm 0.002 \\
(16.0)\end{array}$ \\
\hline 6 & $\begin{array}{c}0.702 \pm 0.005 \\
(7.5)\end{array}$ & $\begin{array}{c}0.704 \pm 0.004 \\
(4.0)\end{array}$ & $\begin{array}{c}0.705 \pm 0.003 \\
(3.0)\end{array}$ & $\begin{array}{c}0.701 \pm 0.004 \\
(9.5)\end{array}$ & $\begin{array}{c}0.706 \pm 0.004 \\
(1.5)\end{array}$ & $\begin{array}{c}0.688 \pm 0.004 \\
(16.0)\end{array}$ \\
\hline 7 & $\begin{array}{c}0.805 \pm 0.002 \\
(13.0)\end{array}$ & $\begin{array}{c}0.802 \pm 0.002 \\
(22.0)\end{array}$ & $\begin{array}{c}0.802 \pm 0.002 \\
(22.0)\end{array}$ & $\begin{array}{c}0.800 \quad 0.002 \\
(24.0)\end{array}$ & $\begin{array}{c}0.803 \pm 0.002 \\
(19.5)\end{array}$ & $\begin{array}{c}0.804 \pm 0.002 \\
(17.0)\end{array}$ \\
\hline 8 & $\begin{array}{c}0.719 \pm 0.005 \\
(6.5)\end{array}$ & $\begin{array}{c}0.718 \pm 0.005 \\
(8.0)\end{array}$ & $\begin{array}{c}0.722 \pm 0.005 \\
(2.0)\end{array}$ & $\begin{array}{c}0.714 \pm 0.006 \\
(9.0)\end{array}$ & $\begin{array}{c}0.722 \pm 0.006 \\
(2.0)\end{array}$ & $\begin{array}{c}0.682 \pm 0.004 \\
(15.5)\end{array}$ \\
\hline 9 & $\begin{array}{c}0.904 \pm 0.002 \\
(9.0)\end{array}$ & $\begin{array}{c}0.905 \pm 0.002 \\
(5.0)\end{array}$ & $\begin{array}{c}0.906 \pm 0.001 \\
(2.0)\end{array}$ & $\begin{array}{c}0.904 \pm 0.001 \\
(9.0)\end{array}$ & $\begin{array}{c}0.906 \pm 0.001 \\
(2.0)\end{array}$ & $\begin{array}{c}0.897 \pm 0.002 \\
(17.0)\end{array}$ \\
\hline 10 & $\begin{array}{c}0.820 \pm 0.005 \\
(2.5)\end{array}$ & $\begin{array}{c}0.819 \pm 0.005 \\
(5.5)\end{array}$ & $\begin{array}{c}0.815 \pm 0.005 \\
(18.5)\end{array}$ & $\begin{array}{c}0.818 \pm 0.005 \\
(10.0)\end{array}$ & $\begin{array}{c}0.818 \pm 0.005 \\
(10.0)\end{array}$ & $\begin{array}{c}0.818 \pm 0.005 \\
(10.0)\end{array}$ \\
\hline 11 & $\begin{array}{c}0.806 \pm 0.002 \\
(3.0)\end{array}$ & $\begin{array}{c}0.806 \pm 0.003 \\
(3.0)\end{array}$ & $\begin{array}{c}0.799 \pm 0.002 \\
(10.5)\end{array}$ & $\begin{array}{c}0.807 \pm 0.002 \\
(1.0)\end{array}$ & $\begin{array}{c}0.796 \pm 0.003 \\
(14.5)\end{array}$ & $\begin{array}{c}0.782 \pm 0.002 \\
\quad(22.5)\end{array}$ \\
\hline 12 & $\begin{array}{c}0.889 \pm 0.004 \\
(2.0)\end{array}$ & $0.887 \pm 0.004$ & $\frac{0.887 \pm 0.003}{(6.5)}$ & $\begin{array}{c}0.885 \pm 0.004 \\
(9.5)\end{array}$ & $\begin{array}{c}0.889 \pm 0.004 \\
(2.0)\end{array}$ & $\begin{array}{c}0.853 \pm 0.004 \\
(16.0)\end{array}$ \\
\hline 13 & $\begin{array}{c}0.922 \pm 0.002 \\
(5.0)\end{array}$ & $\begin{array}{c}0.922 \pm 0.001 \\
(5.0)\end{array}$ & $\begin{array}{c}0.920 \pm 0.001 \\
(13.0)\end{array}$ & $\begin{array}{c}0.921 \pm 0.002 \\
(9.5)\end{array}$ & $\begin{array}{c}0.920 \pm 0.002 \\
(13.0)\end{array}$ & $0.921 \pm 0.001$ \\
\hline 14 & $\begin{array}{c}0.932 \pm 0.004 \\
(7.0)\end{array}$ & $\begin{array}{c}0.934 \pm 0.004 \\
(4.5)\end{array}$ & $\begin{array}{c}0.934 \pm 0.003 \\
(4.5)\end{array}$ & $\begin{array}{c}0.930 \pm 0.004 \\
(9.0)\end{array}$ & $\begin{array}{c}0.935 \pm 0.003 \\
(2.0)\end{array}$ & $\begin{array}{c}0.679 \pm 0.002 \\
(16.0)\end{array}$ \\
\hline 15 & $\begin{array}{c}0.986 \pm 0.001 \\
(12.0)\end{array}$ & $\begin{array}{c}0.986 \pm 0.001 \\
(12.0)\end{array}$ & $\begin{array}{c}0.987 \pm 0.001 \\
(5.0)\end{array}$ & $\begin{array}{c}0.986 \pm 0.001 \\
(12.0)\end{array}$ & $\begin{array}{c}0.987 \pm 0.001 \\
(5.0)\end{array}$ & $\begin{array}{c}0.986 \pm 0.001 \\
(12.0)\end{array}$ \\
\hline 16 & $\begin{array}{c}0.932 \pm 0.004 \\
(7.0)\end{array}$ & $\begin{array}{c}0.935 \pm 0.004 \\
(3.5)\end{array}$ & $0.935 \pm 0.003$ & $\begin{array}{c}0.930 \pm 0.005 \\
(8.5)\end{array}$ & $\begin{array}{c}0.935 \pm 0.003 \\
(3.5)\end{array}$ & $\begin{array}{c}0.678 \pm 0.002 \\
(16.0)\end{array}$ \\
\hline 17 & $\begin{array}{c}0.847 \pm 0.001 \\
(11.5)\end{array}$ & $\begin{array}{c}0.847 \pm 0.001 \\
\quad(11.5)\end{array}$ & $\begin{array}{c}0.846 \pm 0.001 \\
(16.5)\end{array}$ & $\begin{array}{c}0.846 \pm 0.001 \\
(16.5)\end{array}$ & $\begin{array}{c}0.847 \pm 0.001 \\
\quad(11.5)\end{array}$ & $\begin{array}{c}0.851 \pm 0.001 \\
(3.0)\end{array}$ \\
\hline 18 & $\begin{array}{c}0.799 \pm 0.009 \\
(7.5)\end{array}$ & $\begin{array}{c}0.807 \pm 0.009 \\
(5.0)\end{array}$ & $\begin{array}{c}0.823 \pm 0.006 \\
(2.0)\end{array}$ & $\begin{array}{c}0.799 \pm 0.006 \\
(7.5)\end{array}$ & $\begin{array}{c}0.823 \pm 0.007 \\
(2.0)\end{array}$ & $\begin{array}{c}0.565 \pm 0.000 \\
(17.5)\end{array}$ \\
\hline 19 & $\begin{array}{c}0.954 \pm 0.001 \\
(12.0)\end{array}$ & $\begin{array}{c}0.954 \pm 0.001 \\
(12.0)\end{array}$ & $\begin{array}{c}0.954 \pm 0.001 \\
(12.0)\end{array}$ & $\begin{array}{c}0.953 \pm 0.001 \\
(20.5)\end{array}$ & $\begin{array}{c}0.954 \pm 0.001 \\
(12.0)\end{array}$ & $\begin{array}{c}0.951 \pm 0.001 \\
(24.0)\end{array}$ \\
\hline 20 & $\begin{array}{c}0.971 \pm 0.001 \\
(14.5)\end{array}$ & $\begin{array}{c}0.970 \pm 0.001 \\
(20.5)\end{array}$ & $\begin{array}{c}0.970 \pm 0.001 \\
(20.5)\end{array}$ & $\begin{array}{c}0.971 \pm 0.001 \\
(14.5)\end{array}$ & $\begin{array}{c}0.970 \pm 0.001 \\
\quad(20.5)\end{array}$ & $\begin{array}{c}0.973 \pm 0.001 \\
(6.5)\end{array}$ \\
\hline 21 & $\begin{array}{c}0.946 \pm 0.004 \\
(12.0)\end{array}$ & $\begin{array}{c}0.945 \pm 0.006 \\
(14.5)\end{array}$ & $\begin{array}{c}0.941 \pm 0.004 \\
(17.5)\end{array}$ & $\begin{array}{c}0.946 \pm 0.004 \\
(12.0)\end{array}$ & $\begin{array}{c}0.941 \pm 0.004 \\
(17.5)\end{array}$ & $\begin{array}{c}0.982 \pm 0.003 \\
(1.0)\end{array}$ \\
\hline 22 & $\begin{array}{c}0.802 \pm 0.002 \\
(7.5)\end{array}$ & $\begin{array}{c}0.802 \pm 0.002 \\
(7.5)\end{array}$ & $\begin{array}{c}0.803 \pm 0.002 \\
(2.5)\end{array}$ & $\begin{array}{c}0.802 \pm 0.002 \\
(7.5)\end{array}$ & $\begin{array}{c}0.803 \pm 0.002 \\
(2.5)\end{array}$ & $\begin{array}{c}0.799 \pm 0.002 \\
(13.0)\end{array}$ \\
\hline 23 & $\begin{array}{c}0.888 \pm 0.007 \\
(7.0)\end{array}$ & $\begin{array}{c}0.892 \pm 0.007 \\
(4.5)\end{array}$ & $\begin{array}{c}0.897 \pm 0.006 \\
(1.5)\end{array}$ & $\begin{array}{c}0.880 \pm 0.006 \\
(9.0)\end{array}$ & $\begin{array}{c}0.897 \pm 0.007 \\
(1.5)\end{array}$ & $\begin{array}{c}0.563 \pm 0.001 \\
(16.0)\end{array}$ \\
\hline 24 & $\begin{array}{c}0.820 \pm 0.005 \\
(7.0)\end{array}$ & $\begin{array}{c}0.824 \pm 0.004 \\
(5.5)\end{array}$ & $\begin{array}{c}0.840 \pm 0.005 \\
(2.5)\end{array}$ & $\begin{array}{c}0.816 \pm 0.005 \\
(9.0)\end{array}$ & $\begin{array}{c}0.840 \pm 0.005 \\
(2.5)\end{array}$ & $\begin{array}{c}0.794 \pm 0.000 \\
(17.5)\end{array}$ \\
\hline 25 & $\begin{array}{c}0.771 \pm 0.003 \\
(12.5)\end{array}$ & $\begin{array}{c}0.772 \pm 0.004 \\
(9.5)\end{array}$ & $\begin{array}{c}0.771 \pm 0.004 \\
(12.5)\end{array}$ & $\begin{array}{c}0.765 \pm 0.004 \\
(15.0)\end{array}$ & $\begin{array}{c}0.773 \pm 0.003 \\
(7.5)\end{array}$ & $\begin{array}{c}0.781 \pm 0.003 \\
(4.0)\end{array}$ \\
\hline 26 & $\begin{array}{c}0.868 \pm 0.005 \\
(1.5)\end{array}$ & $\begin{array}{c}0.867 \pm 0.004 \\
(3.5)\end{array}$ & $\begin{array}{c}0.864 \pm 0.004 \\
(9.0)\end{array}$ & $\begin{array}{c}0.863 \pm 0.004 \\
(10.0)\end{array}$ & $\begin{array}{c}0.865 \pm 0.005 \\
(7.5)\end{array}$ & $\begin{array}{c}0.858 \pm 0.005 \\
(12.5)\end{array}$ \\
\hline 27 & $\begin{array}{c}1.000 \pm 0.000 \\
(5.0)\end{array}$ & $\begin{array}{c}1.000 \pm 0.000 \\
(5.0)\end{array}$ & $\begin{array}{c}1.000 \pm 0.000 \\
(5.0)\end{array}$ & $\begin{array}{c}1.000 \pm 0.000 \\
(5.0)\end{array}$ & $\begin{array}{c}1.000 \pm 0.000 \\
(5.0)\end{array}$ & $\begin{array}{c}0.987 \pm 0.002 \\
(16.0)\end{array}$ \\
\hline 28 & $\begin{array}{c}0.628 \pm 0.002 \\
(9.0)\end{array}$ & $\begin{array}{c}0.628 \pm 0.002 \\
(9.0)\end{array}$ & $\begin{array}{c}0.627 \pm 0.002 \\
(14.5)\end{array}$ & $\begin{array}{c}0.629 \pm 0.002 \\
(2.5)\end{array}$ & $\begin{array}{c}0.627 \pm 0.002 \\
(14.5)\end{array}$ & $\begin{array}{c}0.629 \pm 0.002 \\
(2.5)\end{array}$ \\
\hline 29 & $\begin{array}{c}0.638 \pm 0.005 \\
(11.5)\end{array}$ & $\begin{array}{c}0.637 \pm 0.004 \\
(15.0)\end{array}$ & $\begin{array}{c}0.637 \pm 0.003 \\
(15.0)\end{array}$ & $\begin{array}{c}0.638 \pm 0.005 \\
(11.5)\end{array}$ & $\begin{array}{c}0.635 \pm 0.004 \\
(20.0)\end{array}$ & $\begin{array}{c}0.641 \pm 0.004 \\
(6.0)\end{array}$ \\
\hline 30 & $0.625 \pm 0.002$ & $0.625 \pm 0.002$ & $0.624 \pm 0.001$ & $0.625 \pm 0.001$ & $0.624 \pm 0.002$ & $0.622 \pm 0.001$ \\
\hline
\end{tabular}




\begin{tabular}{lcccccc}
\hline \hline & $(4.5)$ & $(4.5)$ & $(9.5)$ & $(4.5)$ & $(9.5)$ & $(15.0)$ \\
\hline \hline Rank & $\mathbf{8 . 4 8 3}$ & $\mathbf{8 . 6 3 3}$ & $\mathbf{9 . 8 0 0}$ & $\mathbf{1 0 . 3 8 3}$ & $\mathbf{9 . 3 1 7}$ & $\mathbf{1 3 . 0 5 0}$ \\
\hline \hline
\end{tabular}

Table 4. Testing Accuracies of ELMs Corresponding to 24 Probability Distributions on 30 UCI Data Sets

\begin{tabular}{|c|c|c|c|c|c|c|}
\hline & $\mathrm{D}_{1}$ :Beta & $\mathrm{D}_{2}$ :Cauchy & $\mathrm{D}_{3}:$ Central Chi & $\mathrm{D}_{4}:$ Chi & $\mathrm{D}_{5}$ :Erlang & $\mathrm{D}_{6}:$ Exponential \\
\hline 1 & $\begin{array}{c}0.806 \pm 0.009 \\
(2.5)\end{array}$ & $\begin{array}{c}0.790 \pm 0.012 \\
(23.0)\end{array}$ & $\begin{array}{c}0.803 \pm 0.010 \\
(7.5)\end{array}$ & $0.804 \pm 0.011$ & $0.804 \pm 0.011$ & $\begin{array}{c}0.802 \pm 0.008 \\
(9.0)\end{array}$ \\
\hline 2 & $\begin{array}{c}0.794 \pm 0.004 \\
(10.5)\end{array}$ & $\begin{array}{c}0.790 \pm 0.006 \\
(23.5)\end{array}$ & $0.795 \pm 0.005$ & $\begin{array}{c}0.795 \pm 0.004 \\
(6.5)\end{array}$ & $\begin{array}{c}0.796 \pm 0.005 \\
(3.5)\end{array}$ & $\begin{array}{c}0.792 \pm 0.005 \\
(17.5)\end{array}$ \\
\hline 3 & $\begin{array}{c}0.965 \pm 0.003 \\
(11.0)\end{array}$ & $\begin{array}{c}0.964 \pm 0.004 \\
(16.5)\end{array}$ & $\begin{array}{c}0.968 \pm 0.004 \\
(1.5)\end{array}$ & $\begin{array}{c}0.965 \pm 0.003 \\
(11.0)\end{array}$ & $\begin{array}{c}0.967 \pm 0.003 \\
(5.0)\end{array}$ & $\begin{array}{c}0.966 \pm 0.004 \\
(8.0)\end{array}$ \\
\hline 4 & $\begin{array}{c}0.962 \pm 0.006 \\
(4.5)\end{array}$ & $\begin{array}{c}0.934 \pm 0.009 \\
(12.0)\end{array}$ & $\begin{array}{c}0.823 \pm 0.009 \\
(22.0)\end{array}$ & $\begin{array}{c}0.879 \pm 0.008 \\
(17.0)\end{array}$ & $\begin{array}{c}0.818 \pm 0.010 \\
(23.0)\end{array}$ & $\begin{array}{c}0.913 \pm 0.006 \\
(16.0)\end{array}$ \\
\hline 5 & $\begin{array}{c}0.714 \pm 0.022 \\
(19.5)\end{array}$ & $\begin{array}{c}0.726 \pm 0.025 \\
(18.0)\end{array}$ & $\begin{array}{c}0.743 \pm 0.009 \\
(14.0)\end{array}$ & $\begin{array}{c}0.729 \pm 0.014 \\
(17.0)\end{array}$ & $\begin{array}{c}0.745 \pm 0.007 \\
\quad(12.0)\end{array}$ & $\begin{array}{c}0.704 \pm 0.019 \\
(22.5)\end{array}$ \\
\hline 6 & $\begin{array}{c}0.540 \pm 0.012 \\
(16.0)\end{array}$ & $\begin{array}{c}0.535 \pm 0.013 \\
(18.0)\end{array}$ & $\begin{array}{c}0.533 \pm 0.017 \\
(20.5)\end{array}$ & $\begin{array}{c}0.545 \pm 0.011 \\
(8.5)\end{array}$ & $\begin{array}{c}0.533 \pm 0.014 \\
(20.5)\end{array}$ & $\begin{array}{c}0.540 \pm 0.014 \\
(16.0)\end{array}$ \\
\hline 7 & $\begin{array}{c}0.767 \pm 0.005 \\
(18.0)\end{array}$ & $\begin{array}{c}0.772 \pm 0.009 \\
(8.5)\end{array}$ & $\begin{array}{c}0.774 \pm 0.007 \\
(5.5)\end{array}$ & $\begin{array}{c}0.771 \pm 0.007 \\
(10.0)\end{array}$ & $\begin{array}{c}0.776 \pm 0.008 \\
(1.5)\end{array}$ & $\begin{array}{c}0.772 \pm 0.007 \\
(8.5)\end{array}$ \\
\hline 8 & $\begin{array}{c}0.630 \pm 0.014 \\
(11.0)\end{array}$ & $\begin{array}{c}0.636 \pm 0.018 \\
(9.0)\end{array}$ & $\begin{array}{c}0.588 \pm 0.007 \\
(20.0)\end{array}$ & $\begin{array}{c}0.607 \pm 0.013 \\
(17.0)\end{array}$ & $\begin{array}{c}0.587 \pm 0.006 \\
(21.0)\end{array}$ & $\begin{array}{c}0.612 \pm 0.018 \\
(15.5)\end{array}$ \\
\hline 9 & $0.852 \pm 0.009$ & $\begin{array}{c}0.844 \pm 0.010 \\
(19.0)\end{array}$ & $\begin{array}{c}0.843 \pm 0.010 \\
(20.0)\end{array}$ & $\begin{array}{c}0.850 \pm 0.008 \\
(10.5)\end{array}$ & $\begin{array}{c}0.839 \pm 0.008 \\
(22.0)\end{array}$ & $\begin{array}{c}0.853 \pm 0.008 \\
(3.5)\end{array}$ \\
\hline 10 & $\begin{array}{c}0.647 \pm 0.018 \\
(19.0)\end{array}$ & $\begin{array}{c}0.644 \pm 0.019 \\
(22.0)\end{array}$ & $\begin{array}{c}0.649 \pm 0.023 \\
(14.0)\end{array}$ & $\begin{array}{c}0.657 \pm 0.018 \\
(1.0)\end{array}$ & $\begin{array}{c}0.655 \pm 0.021 \\
(4.0)\end{array}$ & $\begin{array}{c}0.649 \pm 0.018 \\
(14.0)\end{array}$ \\
\hline 11 & $\begin{array}{c}0.734 \pm 0.008 \\
(3.5)\end{array}$ & $\begin{array}{c}0.709 \pm 0.012 \\
(22.0)\end{array}$ & $\begin{array}{c}0.714 \pm 0.012 \\
(13.0)\end{array}$ & $\begin{array}{c}0.723 \pm 0.009 \\
(10.0)\end{array}$ & $\begin{array}{c}0.712 \pm 0.012 \\
\quad(15.5)\end{array}$ & $\begin{array}{c}0.715 \pm 0.010 \\
(11.5)\end{array}$ \\
\hline 12 & $\begin{array}{c}0.801 \pm 0.018 \\
(5.0)\end{array}$ & $\begin{array}{c}0.798 \pm 0.019 \\
(10.5)\end{array}$ & $\begin{array}{c}0.728 \pm 0.021 \\
(22.0)\end{array}$ & $\begin{array}{c}0.748 \pm 0.012 \\
(18.0)\end{array}$ & $\begin{array}{c}0.716 \pm 0.015 \\
\quad(24.0)\end{array}$ & $\begin{array}{c}0.779 \pm 0.019 \\
(13.0)\end{array}$ \\
\hline 13 & $\begin{array}{c}0.923 \pm 0.003 \\
(1.0)\end{array}$ & $\begin{array}{c}0.903 \pm 0.005 \\
(16.0)\end{array}$ & $\begin{array}{c}0.873 \pm 0.006 \\
(20.0)\end{array}$ & $\begin{array}{c}0.902 \pm 0.004 \\
(17.0)\end{array}$ & $\begin{array}{c}0.866 \pm 0.006 \\
(22.0)\end{array}$ & $\begin{array}{c}0.912 \pm 0.003 \\
(15.0)\end{array}$ \\
\hline 14 & $\begin{array}{c}0.803 \pm 0.012 \\
(11.0)\end{array}$ & $\begin{array}{c}0.840 \pm 0.019 \\
(10.0)\end{array}$ & $\begin{array}{c}0.642 \pm 0.001 \\
(21.5)\end{array}$ & $\begin{array}{c}0.649 \pm 0.004 \\
(18.0)\end{array}$ & $\begin{array}{l}0.641 \pm 0.000 \\
(23.5)\end{array}$ & $\begin{array}{c}0.713 \pm 0.012 \\
(13.0)\end{array}$ \\
\hline 15 & $\begin{array}{c}0.953 \pm 0.010 \\
(5.0)\end{array}$ & $\begin{array}{c}0.941 \pm 0.012 \\
(11.0)\end{array}$ & $\begin{array}{c}0.943 \pm 0.012 \\
(8.0)\end{array}$ & $\begin{array}{c}0.945 \pm 0.008 \\
(7.0)\end{array}$ & $\begin{array}{c}0.942 \pm 0.009 \\
(9.5)\end{array}$ & $\begin{array}{c}0.942 \pm 0.011 \\
(9.5)\end{array}$ \\
\hline 16 & $\begin{array}{c}0.805 \pm 0.012 \\
(11.0)\end{array}$ & $\begin{array}{c}0.837 \pm 0.021 \\
(10.0)\end{array}$ & $\begin{array}{c}0.642 \pm 0.002 \\
(21.5)\end{array}$ & $\begin{array}{c}0.649 \pm 0.005 \\
(19.0)\end{array}$ & $\begin{array}{c}0.641 \pm 0.000 \\
(23.5)\end{array}$ & $\begin{array}{c}0.710 \pm 0.012 \\
(13.0)\end{array}$ \\
\hline 17 & $\begin{array}{c}0.842 \pm 0.003 \\
(4.0)\end{array}$ & $\begin{array}{c}0.835 \pm 0.005 \\
(19.0)\end{array}$ & $\begin{array}{c}0.834 \pm 0.004 \\
(20.0)\end{array}$ & $\begin{array}{c}0.838 \pm 0.004 \\
(10.0)\end{array}$ & $\begin{array}{c}0.831 \pm 0.004 \\
\quad(21.0)\end{array}$ & $\begin{array}{c}0.842 \pm 0.003 \\
(4.0)\end{array}$ \\
\hline 18 & $\begin{array}{c}0.565 \pm 0.000 \\
(17.5)\end{array}$ & $\begin{array}{c}0.695 \pm 0.017 \\
(10.0)\end{array}$ & $\begin{array}{c}0.565 \pm 0.000 \\
(17.5)\end{array}$ & $\begin{array}{c}0.565 \pm 0.000 \\
(17.5)\end{array}$ & $\begin{array}{c}0.565 \pm 0.000 \\
(17.5)\end{array}$ & $\begin{array}{c}0.565 \pm 0.000 \\
(17.5)\end{array}$ \\
\hline 19 & $\begin{array}{c}0.939 \pm 0.003 \\
(22.5)\end{array}$ & $\begin{array}{c}0.947 \pm 0.004 \\
(1.0)\end{array}$ & $\begin{array}{c}0.941 \pm 0.003 \\
(10.0)\end{array}$ & $\begin{array}{c}0.940 \pm 0.003 \\
(17.5)\end{array}$ & $\begin{array}{c}0.942 \pm 0.003 \\
(4.5)\end{array}$ & $\begin{array}{c}0.941 \pm 0.003 \\
(10.0)\end{array}$ \\
\hline 20 & $\begin{array}{c}0.932 \pm 0.006 \\
(24.0)\end{array}$ & $\begin{array}{c}0.940 \pm 0.006 \\
(10.5)\end{array}$ & $\begin{array}{c}0.944 \pm 0.005 \\
(2.0)\end{array}$ & $\begin{array}{c}0.943 \pm 0.005 \\
(5.0)\end{array}$ & $\begin{array}{c}0.944 \pm 0.005 \\
(2.0)\end{array}$ & $\begin{array}{c}0.942 \pm 0.004 \\
(8.0)\end{array}$ \\
\hline 21 & $\begin{array}{c}0.903 \pm 0.017 \\
(1.0)\end{array}$ & $\begin{array}{c}0.851 \pm 0.019 \\
(21.0)\end{array}$ & $\begin{array}{c}0.855 \pm 0.018 \\
(19.0)\end{array}$ & $\begin{array}{c}0.876 \pm 0.018 \\
(8.0)\end{array}$ & $\begin{array}{c}0.853 \pm 0.017 \\
(20.0)\end{array}$ & $\begin{array}{c}0.884 \pm 0.020 \\
(6.0)\end{array}$ \\
\hline 22 & $\begin{array}{c}0.767 \pm 0.006 \\
(1.0)\end{array}$ & $\begin{array}{c}0.760 \pm 0.007 \\
(19.0)\end{array}$ & $\begin{array}{c}0.756 \pm 0.008 \\
(21.0)\end{array}$ & $\begin{array}{c}0.766 \pm 0.006 \\
(4.0)\end{array}$ & $\begin{array}{c}0.757 \pm 0.008 \\
(20.0)\end{array}$ & $\begin{array}{c}0.765 \pm 0.007 \\
(9.5)\end{array}$ \\
\hline 23 & $\begin{array}{c}0.698 \pm 0.026 \\
(10.0)\end{array}$ & $\begin{array}{c}0.686 \pm 0.031 \\
(12.0)\end{array}$ & $\begin{array}{c}0.534 \pm 0.000 \\
(22.5)\end{array}$ & $\begin{array}{c}0.539 \pm 0.005 \\
(17.5)\end{array}$ & $\begin{array}{c}0.534 \pm 0.000 \\
\quad(22.5)\end{array}$ & $\begin{array}{c}0.629 \pm 0.017 \\
(13.0)\end{array}$ \\
\hline 24 & $\begin{array}{c}0.790 \pm 0.003 \\
(14.0)\end{array}$ & $\begin{array}{c}0.758 \pm 0.010 \\
(20.0)\end{array}$ & $\begin{array}{c}0.794 \pm 0.000 \\
(7.0)\end{array}$ & $\begin{array}{c}0.794 \pm 0.000 \\
(7.0)\end{array}$ & $\begin{array}{c}0.794 \pm 0.000 \\
(7.0)\end{array}$ & $\begin{array}{c}0.794 \pm 0.000 \\
(7.0)\end{array}$ \\
\hline 25 & $\begin{array}{c}0.734 \pm 0.009 \\
(1.0)\end{array}$ & $\begin{array}{c}0.688 \pm 0.012 \\
(18.0)\end{array}$ & $\begin{array}{c}0.662 \pm 0.012 \\
(20.5)\end{array}$ & $\begin{array}{c}0.703 \pm 0.009 \\
(16.0)\end{array}$ & $\begin{array}{c}0.662 \pm 0.011 \\
\quad(20.5)\end{array}$ & $\begin{array}{c}0.713 \pm 0.010 \\
(14.0)\end{array}$ \\
\hline 26 & $\begin{array}{c}0.783 \pm 0.011 \\
(1.0)\end{array}$ & $\begin{array}{c}0.703 \pm 0.021 \\
(19.0)\end{array}$ & $\begin{array}{c}0.679 \pm 0.016 \\
(20.0)\end{array}$ & $\begin{array}{c}0.759 \pm 0.012 \\
(16.0)\end{array}$ & $\begin{array}{c}0.673 \pm 0.015 \\
\quad(21.0)\end{array}$ & $\begin{array}{c}0.763 \pm 0.013 \\
(15.0)\end{array}$ \\
\hline 27 & $\begin{array}{c}0.971 \pm 0.011 \\
(9.0)\end{array}$ & $\begin{array}{c}0.947 \pm 0.017 \\
(12.5)\end{array}$ & $\begin{array}{c}0.847 \pm 0.020 \\
(20.0)\end{array}$ & $\begin{array}{c}0.908 \pm 0.018 \\
(17.0)\end{array}$ & $\begin{array}{c}0.835 \pm 0.018 \\
(21.0)\end{array}$ & $\begin{array}{c}0.946 \pm 0.014 \\
(14.0)\end{array}$ \\
\hline 28 & $\begin{array}{c}0.598 \pm 0.004 \\
(7.5)\end{array}$ & $\begin{array}{c}0.593 \pm 0.008 \\
(19.5)\end{array}$ & $\begin{array}{c}0.592 \pm 0.008 \\
(21.0)\end{array}$ & $\begin{array}{c}0.599 \pm 0.007 \\
(4.0)\end{array}$ & $\begin{array}{c}0.593 \pm 0.005 \\
(19.5)\end{array}$ & $\begin{array}{c}0.599 \pm 0.006 \\
(4.0)\end{array}$ \\
\hline 29 & $\begin{array}{c}0.527 \pm 0.015 \\
(22.0)\end{array}$ & $\begin{array}{c}0.533 \pm 0.015 \\
(5.5)\end{array}$ & $\begin{array}{c}0.532 \pm 0.015 \\
(10.0)\end{array}$ & $\begin{array}{c}0.535 \pm 0.013 \\
(2.5)\end{array}$ & $\begin{array}{c}0.532 \pm 0.015 \\
(10.0)\end{array}$ & $\begin{array}{c}0.531 \pm 0.013 \\
(14.5)\end{array}$ \\
\hline
\end{tabular}




\begin{tabular}{|c|c|c|c|c|c|c|}
\hline 30 & $\begin{array}{c}0.597 \pm 0.004 \\
(6.5) \\
\end{array}$ & $\begin{array}{c}0.595 \pm 0.005 \\
(14.0) \\
\end{array}$ & $\begin{array}{c}0.590 \pm 0.004 \\
(20.0) \\
\end{array}$ & $\begin{array}{c}0.594 \pm 0.005 \\
(17.0) \\
\end{array}$ & $\begin{array}{c}0.587 \pm 0.004 \\
(21.5) \\
\end{array}$ & $\begin{array}{c}0.596 \pm 0.005 \\
(11.5) \\
\end{array}$ \\
\hline Rank & 9.833 & 15.000 & 15.600 & 11.733 & 15.467 & 11.783 \\
\hline & $\mathrm{D}_{7}: \mathrm{F}$ & $\mathrm{D}_{8}$ :Frechet & $\mathrm{D}_{9}:$ Gamma & $\mathrm{D}_{10}$ :Gumbel & $\mathrm{D}_{11}$ :Hyperbolic & $\mathrm{D}_{12}:$ Laplace \\
\hline 1 & $\begin{array}{c}0.789 \pm 0.013 \\
(24.0)\end{array}$ & $\begin{array}{c}0.806 \pm 0.011 \\
(2.5)\end{array}$ & $\begin{array}{c}0.803 \pm 0.009 \\
(7.5)\end{array}$ & $\begin{array}{c}0.800 \pm 0.010 \\
(12.0)\end{array}$ & $\begin{array}{c}0.796 \pm 0.007 \\
(19.0)\end{array}$ & $\begin{array}{c}0.796 \pm 0.011 \\
(19.0)\end{array}$ \\
\hline 2 & $\begin{array}{c}0.795 \pm 0.005 \\
(6.5)\end{array}$ & $\begin{array}{c}0.796 \pm 0.004 \\
(3.5)\end{array}$ & $\begin{array}{c}0.795 \pm 0.004 \\
(6.5)\end{array}$ & $\begin{array}{c}0.793 \pm 0.005 \\
(13.5)\end{array}$ & $\begin{array}{c}0.792 \pm 0.005 \\
(17.5)\end{array}$ & $\begin{array}{c}0.791 \pm 0.005 \\
(21.5)\end{array}$ \\
\hline 3 & $\begin{array}{c}0.968 \pm 0.003 \\
(1.5)\end{array}$ & $\begin{array}{c}0.964 \pm 0.003 \\
(16.5)\end{array}$ & $\begin{array}{c}0.965 \pm 0.003 \\
\quad(11.0)\end{array}$ & $\begin{array}{c}0.967 \pm 0.004 \\
(5.0)\end{array}$ & $\begin{array}{c}0.964 \pm 0.003 \\
(16.5)\end{array}$ & $\begin{array}{c}0.964 \pm 0.003 \\
(16.5)\end{array}$ \\
\hline 4 & $\begin{array}{c}0.709 \pm 0.039 \\
(24.0)\end{array}$ & $\begin{array}{c}0.916 \pm 0.007 \\
(14.0)\end{array}$ & $\begin{array}{c}0.917 \pm 0.007 \\
(13.0)\end{array}$ & $\begin{array}{c}0.825 \pm 0.011 \\
(20.0)\end{array}$ & $\begin{array}{c}0.962 \pm 0.006 \\
(4.5)\end{array}$ & $\begin{array}{c}0.961 \pm 0.005 \\
(7.5)\end{array}$ \\
\hline 5 & $\begin{array}{c}0.755 \pm 0.006 \\
(9.0)\end{array}$ & $\begin{array}{c}0.704 \pm 0.018 \\
\quad(22.5)\end{array}$ & $\begin{array}{c}0.704 \pm 0.020 \\
\quad(22.5)\end{array}$ & $\begin{array}{c}0.743 \pm 0.012 \\
\quad(14.0)\end{array}$ & $\begin{array}{c}0.760 \pm 0.018 \\
(5.0)\end{array}$ & $\begin{array}{c}0.752 \pm 0.025 \\
(10.0)\end{array}$ \\
\hline 6 & $\begin{array}{c}0.528 \pm 0.012 \\
\quad(24.0)\end{array}$ & $\begin{array}{c}0.543 \pm 0.012 \\
\quad(12.0)\end{array}$ & $\begin{array}{c}0.545 \pm 0.013 \\
(8.5)\end{array}$ & $\begin{array}{l}0.531 \pm 0.013 \\
\quad(22.5)\end{array}$ & $\begin{array}{c}0.545 \pm 0.014 \\
(8.5)\end{array}$ & $\begin{array}{c}0.546 \pm 0.013 \\
(5.5)\end{array}$ \\
\hline 7 & $\begin{array}{c}0.775 \pm 0.008 \\
(3.0)\end{array}$ & $\begin{array}{c}0.768 \pm 0.006 \\
(15.0)\end{array}$ & $\begin{array}{c}0.769 \pm 0.008 \\
(12.0)\end{array}$ & $\begin{array}{c}0.774 \pm 0.008 \\
(5.5)\end{array}$ & $\begin{array}{c}0.765 \pm 0.007 \\
(21.0)\end{array}$ & $\begin{array}{c}0.770 \pm 0.008 \\
(11.0)\end{array}$ \\
\hline 8 & $\begin{array}{c}0.585 \pm 0.005 \\
(23.0)\end{array}$ & $\begin{array}{c}0.614 \pm 0.013 \\
(14.0)\end{array}$ & $\begin{array}{c}0.612 \pm 0.014 \\
\quad(15.5)\end{array}$ & $\begin{array}{c}0.586 \pm 0.008 \\
\quad(22.0)\end{array}$ & $\begin{array}{c}0.647 \pm 0.014 \\
(3.5)\end{array}$ & $\begin{array}{c}0.645 \pm 0.019 \\
(7.0)\end{array}$ \\
\hline 9 & $\begin{array}{c}0.836 \pm 0.010 \\
(24.0)\end{array}$ & $\begin{array}{c}0.851 \pm 0.007 \\
(7.5)\end{array}$ & $\begin{array}{c}0.853 \pm 0.007 \\
(3.5)\end{array}$ & $\begin{array}{c}0.838 \pm 0.009 \\
(23.0)\end{array}$ & $\begin{array}{c}0.849 \pm 0.008 \\
(13.5)\end{array}$ & $\begin{array}{c}0.849 \pm 0.010 \\
(13.5)\end{array}$ \\
\hline 10 & $\begin{array}{c}0.643 \pm 0.019 \\
(23.0)\end{array}$ & $\begin{array}{c}0.656 \pm 0.017 \\
(2.0)\end{array}$ & $\begin{array}{c}0.650 \pm 0.019 \\
(9.5)\end{array}$ & $\begin{array}{c}0.641 \pm 0.023 \\
(24.0)\end{array}$ & $\begin{array}{c}0.649 \pm 0.017 \\
(14.0)\end{array}$ & $\begin{array}{c}0.655 \pm 0.017 \\
(4.0)\end{array}$ \\
\hline 11 & $\begin{array}{c}0.710 \pm 0.010 \\
(19.5)\end{array}$ & $\begin{array}{c}0.734 \pm 0.007 \\
(3.5)\end{array}$ & $\begin{array}{c}0.735 \pm 0.008 \\
(2.0)\end{array}$ & $\begin{array}{c}0.707 \pm 0.011 \\
\quad(24.0)\end{array}$ & $\begin{array}{c}0.712 \pm 0.011 \\
\quad(15.5)\end{array}$ & $\begin{array}{c}0.710 \pm 0.014 \\
(19.5)\end{array}$ \\
\hline 12 & $\begin{array}{c}0.729 \pm 0.020 \\
(21.0)\end{array}$ & $\begin{array}{c}0.775 \pm 0.016 \\
\quad(15.0)\end{array}$ & $\begin{array}{c}0.778 \pm 0.014 \\
\quad(14.0)\end{array}$ & $\begin{array}{c}0.734 \pm 0.016 \\
(20.0)\end{array}$ & $\begin{array}{c}0.803 \pm 0.014 \\
(2.5)\end{array}$ & $\begin{array}{c}0.800 \pm 0.017 \\
(8.0)\end{array}$ \\
\hline 13 & $\begin{array}{c}0.797 \pm 0.011 \\
\quad(24.0)\end{array}$ & $\begin{array}{c}0.916 \pm 0.003 \\
(4.5)\end{array}$ & $\begin{array}{c}0.914 \pm 0.002 \\
(12.5)\end{array}$ & $\begin{array}{c}0.858 \pm 0.006 \\
\quad(23.0)\end{array}$ & $\begin{array}{c}0.915 \pm 0.003 \\
(8.5)\end{array}$ & $\begin{array}{c}0.915 \pm 0.004 \\
(8.5)\end{array}$ \\
\hline 14 & $\begin{array}{c}0.642 \pm 0.002 \\
(21.5)\end{array}$ & $\begin{array}{c}0.691 \pm 0.010 \\
(14.0)\end{array}$ & $\begin{array}{c}0.687 \pm 0.008 \\
\quad(15.0)\end{array}$ & $\begin{array}{l}0.648 \pm 0.005 \\
\quad(19.0)\end{array}$ & $\begin{array}{c}0.884 \pm 0.014 \\
(2.0)\end{array}$ & $\begin{array}{c}0.874 \pm 0.015 \\
(9.0)\end{array}$ \\
\hline 15 & $\begin{array}{c}0.937 \pm 0.010 \\
(14.0)\end{array}$ & $\begin{array}{c}0.955 \pm 0.008 \\
(3.5)\end{array}$ & $\begin{array}{c}0.955 \pm 0.008 \\
(3.5)\end{array}$ & $\begin{array}{c}0.940 \pm 0.010 \\
(12.5)\end{array}$ & $\begin{array}{c}0.935 \pm 0.013 \\
(21.0)\end{array}$ & $\begin{array}{c}0.935 \pm 0.011 \\
(21.0)\end{array}$ \\
\hline 16 & $\begin{array}{c}0.642 \pm 0.002 \\
(21.5)\end{array}$ & $\begin{array}{c}0.693 \pm 0.009 \\
(14.0)\end{array}$ & $\begin{array}{c}0.686 \pm 0.008 \\
(15.0)\end{array}$ & $\begin{array}{c}0.650 \pm 0.005 \\
(18.0)\end{array}$ & $\begin{array}{c}0.881 \pm 0.012 \\
(5.0)\end{array}$ & $\begin{array}{c}0.878 \pm 0.013 \\
(8.0)\end{array}$ \\
\hline 17 & $\begin{array}{c}0.824 \pm 0.006 \\
(24.0)\end{array}$ & $\begin{array}{c}0.842 \pm 0.004 \\
(4.0)\end{array}$ & $\begin{array}{c}0.842 \pm 0.004 \\
(4.0)\end{array}$ & $\begin{array}{c}0.830 \pm 0.005 \\
(22.0)\end{array}$ & $\begin{array}{c}0.838 \pm 0.003 \\
(10.0)\end{array}$ & $\begin{array}{c}0.837 \pm 0.004 \\
(15.5)\end{array}$ \\
\hline 18 & $\begin{array}{c}0.565 \pm 0.000 \\
(17.5)\end{array}$ & $\begin{array}{c}0.565 \pm 0.000 \\
(17.5)\end{array}$ & $\begin{array}{c}0.565 \pm 0.000 \\
(17.5)\end{array}$ & $\begin{array}{c}0.565 \pm 0.000 \\
(17.5)\end{array}$ & $\begin{array}{c}0.749 \pm 0.018 \\
(4.5)\end{array}$ & $\begin{array}{c}0.735 \pm 0.021 \\
(9.0)\end{array}$ \\
\hline 19 & $\begin{array}{c}0.946 \pm 0.004 \\
(2.0)\end{array}$ & $\begin{array}{c}0.939 \pm 0.003 \\
(22.5)\end{array}$ & $\begin{array}{c}0.940 \pm 0.003 \\
(17.5)\end{array}$ & $\begin{array}{c}0.942 \pm 0.003 \\
(4.5)\end{array}$ & $\begin{array}{c}0.941 \pm 0.003 \\
(10.0)\end{array}$ & $\begin{array}{c}0.940 \pm 0.003 \\
(17.5)\end{array}$ \\
\hline 20 & $\begin{array}{c}0.942 \pm 0.005 \\
(8.0)\end{array}$ & $\begin{array}{c}0.940 \pm 0.005 \\
(10.5)\end{array}$ & $\begin{array}{c}0.939 \pm 0.004 \\
(12.0)\end{array}$ & $\begin{array}{c}0.943 \pm 0.005 \\
(5.0)\end{array}$ & $\begin{array}{c}0.935 \pm 0.005 \\
(20.5)\end{array}$ & $\begin{array}{c}0.935 \pm 0.006 \\
(20.5)\end{array}$ \\
\hline 21 & $\begin{array}{c}0.816 \pm 0.019 \\
(24.0)\end{array}$ & $\begin{array}{c}0.894 \pm 0.016 \\
(5.0)\end{array}$ & $\begin{array}{c}0.895 \pm 0.016 \\
(3.5)\end{array}$ & $\begin{array}{c}0.846 \pm 0.020 \\
(23.0)\end{array}$ & $\begin{array}{c}0.869 \pm 0.015 \\
(15.0)\end{array}$ & $\begin{array}{c}0.872 \pm 0.018 \\
(10.5)\end{array}$ \\
\hline 22 & $\begin{array}{c}0.752 \pm 0.009 \\
\quad(24.0)\end{array}$ & $\begin{array}{c}0.765 \pm 0.006 \\
(9.5)\end{array}$ & $\begin{array}{c}0.765 \pm 0.006 \\
(9.5)\end{array}$ & $\begin{array}{c}0.755 \pm 0.010 \\
(22.5)\end{array}$ & $\begin{array}{c}0.765 \pm 0.008 \\
(9.5)\end{array}$ & $\begin{array}{c}0.763 \pm 0.007 \\
(15.5)\end{array}$ \\
\hline 23 & $\begin{array}{c}0.534 \pm 0.000 \\
(22.5)\end{array}$ & $\begin{array}{c}0.592 \pm 0.013 \\
\quad(14.0)\end{array}$ & $\begin{array}{c}0.572 \pm 0.009 \\
(15.0)\end{array}$ & $\begin{array}{c}0.537 \pm 0.005 \\
(19.0)\end{array}$ & $\begin{array}{c}0.757 \pm 0.025 \\
(4.0)\end{array}$ & $\begin{array}{c}0.748 \pm 0.028 \\
(8.0)\end{array}$ \\
\hline 24 & $\begin{array}{c}0.794 \pm 0.000 \\
(7.0)\end{array}$ & $\begin{array}{c}0.794 \pm 0.000 \\
(7.0)\end{array}$ & $\begin{array}{c}0.794 \pm 0.000 \\
(7.0)\end{array}$ & $\begin{array}{c}0.794 \pm 0.000 \\
(7.0)\end{array}$ & $\begin{array}{c}0.757 \pm 0.016 \\
\quad(21.0)\end{array}$ & $\begin{array}{c}0.749 \pm 0.017 \\
(24.0)\end{array}$ \\
\hline 25 & $\begin{array}{c}0.613 \pm 0.016 \\
(24.0)\end{array}$ & $\begin{array}{c}0.721 \pm 0.009 \\
(3.5)\end{array}$ & $\begin{array}{c}0.719 \pm 0.011 \\
(6.0)\end{array}$ & $\begin{array}{c}0.648 \pm 0.015 \\
\quad(23.0)\end{array}$ & $\begin{array}{c}0.720 \pm 0.010 \\
(5.0)\end{array}$ & $\begin{array}{c}0.716 \pm 0.011 \\
(8.5)\end{array}$ \\
\hline 26 & $\begin{array}{c}0.528 \pm 0.020 \\
(24.0)\end{array}$ & $\begin{array}{c}0.776 \pm 0.014 \\
(12.5)\end{array}$ & $\begin{array}{c}0.780 \pm 0.013 \\
(3.0)\end{array}$ & $\begin{array}{c}0.631 \pm 0.018 \\
\quad(23.0)\end{array}$ & $\begin{array}{c}0.779 \pm 0.013 \\
(5.5)\end{array}$ & $\begin{array}{c}0.777 \pm 0.012 \\
(10.5)\end{array}$ \\
\hline 27 & $\begin{array}{c}0.808 \pm 0.027 \\
(24.0)\end{array}$ & $\begin{array}{c}0.944 \pm 0.011 \\
(15.0)\end{array}$ & $\begin{array}{c}0.947 \pm 0.015 \\
(12.5)\end{array}$ & $\begin{array}{c}0.832 \pm 0.021 \\
(22.5)\end{array}$ & $\begin{array}{c}0.975 \pm 0.012 \\
(3.0)\end{array}$ & $\begin{array}{c}0.975 \pm 0.012 \\
(3.0)\end{array}$ \\
\hline 28 & $\begin{array}{c}0.579 \pm 0.008 \\
(24.0)\end{array}$ & $\begin{array}{c}0.599 \pm 0.008 \\
(4.0)\end{array}$ & $\begin{array}{c}0.601 \pm 0.006 \\
(1.0)\end{array}$ & $\begin{array}{c}0.588 \pm 0.008 \\
(23.0)\end{array}$ & $\begin{array}{c}0.597 \pm 0.007 \\
(10.5)\end{array}$ & $\begin{array}{c}0.597 \pm 0.006 \\
(10.5)\end{array}$ \\
\hline 29 & $\begin{array}{c}0.519 \pm 0.015 \\
(24.0)\end{array}$ & $0.533 \pm 0.011$ & $\begin{array}{c}0.534 \pm 0.013 \\
(4.0)\end{array}$ & $\begin{array}{c}0.527 \pm 0.017 \\
\quad(22.0)\end{array}$ & $\begin{array}{c}0.532 \pm 0.014 \\
(10.0)\end{array}$ & $\begin{array}{c}0.530 \pm 0.016 \\
(16.5)\end{array}$ \\
\hline 30 & $\begin{array}{c}0.579 \pm 0.005 \\
(24.0) \\
\end{array}$ & $\begin{array}{c}0.595 \pm 0.004 \\
(14.0) \\
\end{array}$ & $\begin{array}{c}0.594 \pm 0.004 \\
(17.0) \\
\end{array}$ & $\begin{array}{c}0.587 \pm 0.005 \\
(21.5) \\
\end{array}$ & $0.597 \pm 0.004(6.5)$ & $\begin{array}{c}0.598 \pm 0.005 \\
(1.5)\end{array}$ \\
\hline
\end{tabular}




\begin{tabular}{|c|c|c|c|c|c|c|}
\hline Rank & 18.550 & 10.283 & 10.033 & 17.783 & 10.417 & 12.017 \\
\hline & $\mathrm{D}_{13}$ :Logistic & $\mathrm{D}_{14}$ :Lognormal & $\mathrm{D}_{15}$ :Normal & $\mathrm{D}_{16}$ :Pareto & $\mathrm{D}_{17}:$ Rayleigh & $\mathrm{D}_{18}$ :Semicircle \\
\hline 1 & $\begin{array}{c}0.801 \pm 0.009 \\
(10.5)\end{array}$ & $\begin{array}{c}0.799 \pm 0.009 \\
(13.0)\end{array}$ & $\begin{array}{c}0.796 \pm 0.010 \\
(19.0)\end{array}$ & $\begin{array}{c}0.801 \pm 0.007 \\
(10.5)\end{array}$ & $\begin{array}{c}0.805 \pm 0.010 \\
(4.0)\end{array}$ & $\begin{array}{c}0.798 \pm 0.008 \\
\quad(14.0)\end{array}$ \\
\hline 2 & $\begin{array}{c}0.794 \pm 0.005 \\
(10.5)\end{array}$ & $\begin{array}{c}0.793 \pm 0.005 \\
(13.5)\end{array}$ & $\begin{array}{c}0.792 \pm 0.004 \\
(17.5)\end{array}$ & $\begin{array}{c}0.797 \pm 0.005 \\
(2.0)\end{array}$ & $\begin{array}{c}0.794 \pm 0.005 \\
(10.5)\end{array}$ & $\begin{array}{c}0.792 \pm 0.004 \\
\quad(17.5)\end{array}$ \\
\hline 3 & $\begin{array}{c}0.967 \pm 0.004 \\
(5.0)\end{array}$ & $\begin{array}{c}0.967 \pm 0.003 \\
(5.0)\end{array}$ & $\begin{array}{c}0.963 \pm 0.004 \\
(22.0)\end{array}$ & $\begin{array}{c}0.967 \pm 0.003 \\
(5.0)\end{array}$ & $\begin{array}{c}0.965 \pm 0.003 \\
(11.0)\end{array}$ & $\begin{array}{c}0.963 \pm 0.003 \\
(22.0)\end{array}$ \\
\hline 4 & $\begin{array}{c}0.824 \pm 0.009 \\
(21.0)\end{array}$ & $\begin{array}{c}0.857 \pm 0.010 \\
(18.0)\end{array}$ & $\begin{array}{c}0.963 \pm 0.004 \\
(1.5)\end{array}$ & $\begin{array}{c}0.854 \pm 0.008 \\
(19.0)\end{array}$ & $\begin{array}{c}0.949 \pm 0.008 \\
(11.0)\end{array}$ & $\begin{array}{c}0.960 \pm 0.005 \\
(9.5)\end{array}$ \\
\hline 5 & $\begin{array}{c}0.743 \pm 0.011 \\
(14.0)\end{array}$ & $\begin{array}{c}0.733 \pm 0.013 \\
(16.0)\end{array}$ & $\begin{array}{c}0.759 \pm 0.019 \\
(6.5)\end{array}$ & $\begin{array}{c}0.748 \pm 0.009 \\
(11.0)\end{array}$ & $\begin{array}{c}0.704 \pm 0.018 \\
\quad(22.5)\end{array}$ & $\begin{array}{c}0.762 \pm 0.021 \\
(3.5)\end{array}$ \\
\hline 6 & $\begin{array}{c}0.531 \pm 0.012 \\
(22.5)\end{array}$ & $\begin{array}{c}0.534 \pm 0.017 \\
(19.0)\end{array}$ & $\begin{array}{c}0.541 \pm 0.013 \\
(14.0)\end{array}$ & $\begin{array}{c}0.542 \pm 0.013 \\
\quad(13.0)\end{array}$ & $\begin{array}{c}0.545 \pm 0.015 \\
(8.5)\end{array}$ & $\begin{array}{c}0.550 \pm 0.011 \\
(1.5)\end{array}$ \\
\hline 7 & $\begin{array}{c}0.774 \pm 0.008 \\
(5.5)\end{array}$ & $\begin{array}{c}0.776 \pm 0.008 \\
(1.5)\end{array}$ & $\begin{array}{c}0.766 \pm 0.008 \\
(19.0)\end{array}$ & $\begin{array}{c}0.774 \pm 0.008 \\
(5.5)\end{array}$ & $\begin{array}{c}0.768 \pm 0.007 \\
(15.0)\end{array}$ & $\begin{array}{c}0.768 \pm 0.006 \\
\quad(15.0)\end{array}$ \\
\hline 8 & $\begin{array}{c}0.584 \pm 0.008 \\
\quad(24.0)\end{array}$ & $\begin{array}{c}0.596 \pm 0.013 \\
(18.0)\end{array}$ & $0.646 \pm 0.013$ & $\begin{array}{c}0.589 \pm 0.007 \\
(19.0)\end{array}$ & $\begin{array}{c}0.629 \pm 0.014 \\
(12.0)\end{array}$ & $\begin{array}{c}0.643 \pm 0.016 \\
(8.0)\end{array}$ \\
\hline 9 & $\begin{array}{c}0.842 \pm 0.010 \\
\quad(21.0)\end{array}$ & $\begin{array}{c}0.847 \pm 0.010 \\
\quad(16.0)\end{array}$ & $\begin{array}{c}0.850 \pm 0.011 \\
(10.5)\end{array}$ & $\begin{array}{c}0.854 \pm 0.009 \\
(2.0)\end{array}$ & $\begin{array}{c}0.850 \pm 0.008 \\
(10.5)\end{array}$ & $\begin{array}{c}0.847 \pm 0.010 \\
(16.0)\end{array}$ \\
\hline 10 & $\begin{array}{c}0.649 \quad 0.023 \\
(14.0)\end{array}$ & $\begin{array}{c}0.649 \pm 0.019 \\
(14.0)\end{array}$ & $\begin{array}{c}0.646 \pm 0.016 \\
\quad(21.0)\end{array}$ & $\begin{array}{c}0.649 \pm 0.020 \\
(14.0)\end{array}$ & $\begin{array}{c}0.650 \pm 0.023 \\
(9.5)\end{array}$ & $\begin{array}{c}0.649 \pm 0.021 \\
\quad(14.0)\end{array}$ \\
\hline 11 & $\begin{array}{c}0.713 \pm 0.010 \\
(14.0)\end{array}$ & $\begin{array}{c}0.715 \pm 0.010 \\
(11.5)\end{array}$ & $\begin{array}{c}0.710 \pm 0.009 \\
(19.5)\end{array}$ & $\begin{array}{c}0.729 \pm 0.009 \\
(6.0)\end{array}$ & $\begin{array}{c}0.732 \pm 0.011 \\
(5.0)\end{array}$ & $\begin{array}{c}0.725 \pm 0.010 \\
(7.0)\end{array}$ \\
\hline 12 & $\begin{array}{c}0.722 \pm 0.017 \\
\quad(23.0)\end{array}$ & $\begin{array}{c}0.753 \pm 0.017 \\
(17.0)\end{array}$ & $\begin{array}{c}0.803 \pm 0.017 \\
(2.5)\end{array}$ & $\begin{array}{c}0.740 \pm 0.017 \\
(19.0)\end{array}$ & $\begin{array}{c}0.797 \pm 0.016 \\
(12.0)\end{array}$ & $\begin{array}{c}0.801 \pm 0.014 \\
(5.0)\end{array}$ \\
\hline 13 & $\begin{array}{c}0.869 \pm 0.006 \\
(21.0)\end{array}$ & $\begin{array}{c}0.894 \pm 0.004 \\
(18.0)\end{array}$ & $\begin{array}{c}0.915 \pm 0.003 \\
(8.5)\end{array}$ & $\begin{array}{c}0.892 \pm 0.003 \\
(19.0)\end{array}$ & $\begin{array}{c}0.921 \pm 0.003 \\
(2.0)\end{array}$ & $\begin{array}{c}0.914 \pm 0.003 \\
(12.5)\end{array}$ \\
\hline 14 & $\begin{array}{c}0.644 \pm 0.004 \\
(20.0)\end{array}$ & $\begin{array}{c}0.655 \pm 0.006 \\
(17.0)\end{array}$ & $\begin{array}{c}0.885 \pm 0.014 \\
(1.0)\end{array}$ & $\begin{array}{c}0.641 \pm 0.000 \\
(23.5)\end{array}$ & $\begin{array}{c}0.797 \pm 0.010 \\
(12.0)\end{array}$ & $\begin{array}{c}0.879 \pm 0.013 \\
(5.5)\end{array}$ \\
\hline 15 & $\begin{array}{c}0.936 \pm 0.011 \\
(17.0)\end{array}$ & $\begin{array}{c}0.940 \pm 0.010 \\
(12.5)\end{array}$ & $\begin{array}{c}0.935 \pm 0.011 \\
(21.0)\end{array}$ & $\begin{array}{c}0.958 \pm 0.010 \\
(2.0)\end{array}$ & $\begin{array}{c}0.951 \pm 0.009 \\
(6.0)\end{array}$ & $\begin{array}{c}0.936 \pm 0.013 \\
(17.0)\end{array}$ \\
\hline 16 & $\begin{array}{c}0.645 \pm 0.004 \\
(20.0)\end{array}$ & $\begin{array}{c}0.655 \pm 0.006 \\
(17.0)\end{array}$ & $\begin{array}{c}0.882 \pm 0.012 \\
(2.5)\end{array}$ & $\begin{array}{c}0.641 \pm 0.000 \\
(23.5)\end{array}$ & $\begin{array}{c}0.796 \pm 0.012 \\
(12.0)\end{array}$ & $\begin{array}{c}0.881 \pm 0.014 \\
(5.0)\end{array}$ \\
\hline 17 & $\begin{array}{c}0.828 \pm 0.005 \\
\quad(23.0)\end{array}$ & $\begin{array}{c}0.839 \pm 0.005 \\
(7.0)\end{array}$ & $\begin{array}{c}0.837 \pm 0.004 \\
(15.5)\end{array}$ & $\begin{array}{c}0.837 \pm 0.004 \\
(15.5)\end{array}$ & $\begin{array}{c}0.843 \pm 0.003 \\
(1.0)\end{array}$ & $\begin{array}{c}0.838 \pm 0.003 \\
(10.0)\end{array}$ \\
\hline 18 & $\begin{array}{c}0.565 \pm 0.000 \\
(17.5)\end{array}$ & $\begin{array}{c}0.565 \pm 0.000 \\
(17.5)\end{array}$ & $\begin{array}{c}0.746 \pm 0.016 \\
(6.0)\end{array}$ & $\begin{array}{c}0.565 \pm 0.000 \\
(17.5)\end{array}$ & $\begin{array}{c}0.565 \pm 0.000 \\
(17.5)\end{array}$ & $\begin{array}{c}0.763 \pm 0.018 \\
(1.0)\end{array}$ \\
\hline 19 & $\begin{array}{c}0.941 \pm 0.004 \\
(10.0)\end{array}$ & $\begin{array}{c}0.943 \pm 0.003 \\
(3.0)\end{array}$ & $\begin{array}{c}0.941 \pm 0.003 \\
(10.0)\end{array}$ & $\begin{array}{l}0.941 \pm 0.004 \\
\quad(10.0)\end{array}$ & $\begin{array}{c}0.939 \pm 0.003 \\
(22.5)\end{array}$ & $\begin{array}{c}0.941 \pm 0.004 \\
(10.0)\end{array}$ \\
\hline 20 & $\begin{array}{c}0.943 \pm 0.005 \\
(5.0)\end{array}$ & $\begin{array}{c}0.944 \pm 0.005 \\
(2.0)\end{array}$ & $\begin{array}{c}0.936 \pm 0.005 \\
(16.0)\end{array}$ & $\begin{array}{c}0.942 \pm 0.005 \\
(8.0)\end{array}$ & $\begin{array}{c}0.936 \pm 0.005 \\
(16.0)\end{array}$ & $\begin{array}{c}0.935 \pm 0.007 \\
(20.5)\end{array}$ \\
\hline 21 & $\begin{array}{c}0.850 \pm 0.020 \\
(22.0)\end{array}$ & $\begin{array}{c}0.864 \pm 0.017 \\
(18.0)\end{array}$ & $\begin{array}{c}0.868 \pm 0.016 \\
(16.5)\end{array}$ & $\begin{array}{c}0.880 \pm 0.016 \\
(7.0)\end{array}$ & $\begin{array}{c}0.896 \pm 0.018 \\
(2.0)\end{array}$ & $\begin{array}{c}0.870 \pm 0.016 \\
(14.0)\end{array}$ \\
\hline 22 & $\begin{array}{c}0.755 \pm 0.009 \\
(22.5)\end{array}$ & $\begin{array}{c}0.761 \pm 0.007 \\
(18.0)\end{array}$ & $\begin{array}{c}0.766 \pm 0.007 \\
(4.0)\end{array}$ & $\begin{array}{c}0.762 \pm 0.005 \\
(17.0)\end{array}$ & $\begin{array}{c}0.766 \pm 0.007 \\
(4.0)\end{array}$ & $\begin{array}{c}0.766 \pm 0.007 \\
(4.0)\end{array}$ \\
\hline 23 & $\begin{array}{c}0.536 \pm 0.003 \\
(20.0)\end{array}$ & $\begin{array}{c}0.539 \pm 0.006 \\
(17.5)\end{array}$ & $\begin{array}{c}0.754 \pm 0.023 \\
(7.0)\end{array}$ & $\begin{array}{c}0.534 \pm 0.000 \\
(22.5)\end{array}$ & $\begin{array}{c}0.689 \pm 0.021 \\
(11.0)\end{array}$ & $\begin{array}{c}0.759 \pm 0.025 \\
(2.0)\end{array}$ \\
\hline 24 & $\begin{array}{c}0.794 \pm 0.000 \\
(7.0)\end{array}$ & $\begin{array}{c}0.794 \pm 0.000 \\
(7.0)\end{array}$ & $\begin{array}{c}0.759 \pm 0.016 \\
(19.0)\end{array}$ & $\begin{array}{c}0.794 \pm 0.000 \\
(7.0)\end{array}$ & $\begin{array}{c}0.794 \pm 0.001 \\
(7.0)\end{array}$ & $\begin{array}{c}0.773 \pm 0.015 \\
\quad(15.5)\end{array}$ \\
\hline 25 & $\begin{array}{c}0.650 \pm 0.012 \\
(22.0)\end{array}$ & $\begin{array}{c}0.687 \pm 0.013 \\
\quad(19.0)\end{array}$ & $\begin{array}{c}0.716 \pm 0.010 \\
(8.5)\end{array}$ & $\begin{array}{c}0.696 \pm 0.009 \\
(17.0)\end{array}$ & $\begin{array}{c}0.727 \pm 0.008 \\
(2.0)\end{array}$ & $\begin{array}{c}0.714 \pm 0.010 \\
(13.0)\end{array}$ \\
\hline 26 & $\begin{array}{c}0.658 \pm 0.018 \\
(22.0)\end{array}$ & $\begin{array}{c}0.724 \pm 0.015 \\
\quad(18.0)\end{array}$ & $\begin{array}{c}0.778 \pm 0.013 \\
(8.0)\end{array}$ & $\begin{array}{c}0.755 \pm 0.014 \\
\quad(17.0)\end{array}$ & $\begin{array}{c}0.778 \pm 0.011 \\
(8.0)\end{array}$ & $\begin{array}{c}0.777 \pm 0.011 \\
\quad(10.5)\end{array}$ \\
\hline 27 & $\begin{array}{c}0.832 \pm 0.022 \\
(22.5)\end{array}$ & $\begin{array}{c}0.889 \pm 0.016 \\
\quad(18.0)\end{array}$ & $\begin{array}{c}0.975 \pm 0.011 \\
(3.0)\end{array}$ & $\begin{array}{c}0.887 \pm 0.018 \\
\quad(19.0)\end{array}$ & $\begin{array}{c}0.969 \pm 0.011 \\
(11.0)\end{array}$ & $\begin{array}{c}0.972 \pm 0.009 \\
(7.5)\end{array}$ \\
\hline 28 & $\begin{array}{c}0.591 \pm 0.007 \\
(22.0)\end{array}$ & $\begin{array}{c}0.594 \pm 0.007 \\
(17.5)\end{array}$ & $\begin{array}{c}0.597 \pm 0.007 \\
(10.5)\end{array}$ & $\begin{array}{c}0.596 \pm 0.006 \\
(14.5)\end{array}$ & $\begin{array}{c}0.599 \pm 0.005 \\
(4.0)\end{array}$ & $\begin{array}{c}0.594 \pm 0.006 \\
(17.5)\end{array}$ \\
\hline 29 & $\begin{array}{c}0.528 \pm 0.014 \\
\quad(20.0)\end{array}$ & $\begin{array}{c}0.535 \pm 0.012 \\
(2.5)\end{array}$ & $\begin{array}{c}0.529 \pm 0.009 \\
(18.5)\end{array}$ & $\begin{array}{c}0.541 \pm 0.015 \\
(1.0)\end{array}$ & $\begin{array}{c}0.527 \pm 0.014 \\
(22.0)\end{array}$ & $\begin{array}{c}0.530 \pm 0.011 \\
(16.5)\end{array}$ \\
\hline 30 & $\begin{array}{c}0.586 \pm 0.005 \\
(23.0) \\
\end{array}$ & $\begin{array}{c}0.595 \pm 0.005 \\
(14.0) \\
\end{array}$ & $\begin{array}{c}0.597 \pm 0.005 \\
(6.5) \\
\end{array}$ & $\begin{array}{c}0.592 \pm 0.005 \\
(19.0) \\
\end{array}$ & $\begin{array}{c}0.596 \pm 0.005 \\
(11.5) \\
\end{array}$ & $\begin{array}{c}0.597 \pm 0.004 \\
(6.5) \\
\end{array}$ \\
\hline Rank & 17.383 & 13.533 & 11.350 & $\begin{array}{l}12.867 \\
\end{array}$ & 10.100 & 10.717 \\
\hline & $\mathrm{D}_{19}$ :Student ${ }_{i}^{-} \mathrm{s}-\mathrm{t}$ & $\mathrm{D}_{20}$ :Triangular & $\mathrm{D}_{21}$ :Truncated & $\mathrm{D}_{22}$ :Uniform & $\mathrm{D}_{23}$ :Von Mis & $\mathrm{D}_{24}$ :Weibull \\
\hline
\end{tabular}




\begin{tabular}{|c|c|c|c|c|c|c|}
\hline 1 & $\begin{array}{c}0.795 \pm 0.011 \\
(22.0)\end{array}$ & $\begin{array}{c}0.796 \pm 0.011 \\
(19.0)\end{array}$ & $\begin{array}{c}0.797 \pm 0.010 \\
(15.5)\end{array}$ & $\begin{array}{c}0.796 \pm 0.010 \\
(19.0)\end{array}$ & $\begin{array}{c}0.797 \pm 0.012 \\
(15.5)\end{array}$ & $\begin{array}{c}0.808 \pm 0.008 \\
(1.0)\end{array}$ \\
\hline 2 & $\begin{array}{c}0.791 \pm 0.004 \\
\quad(21.5)\end{array}$ & $\begin{array}{c}0.792 \pm 0.004 \\
(17.5)\end{array}$ & $\begin{array}{c}0.794 \pm 0.005 \\
(10.5)\end{array}$ & $\begin{array}{c}0.790 \pm 0.005 \\
(23.5)\end{array}$ & $\begin{array}{c}0.792 \pm 0.004 \\
(17.5)\end{array}$ & $\begin{array}{c}0.798 \pm 0.003 \\
(1.0)\end{array}$ \\
\hline 3 & $\begin{array}{c}0.964 \pm 0.003 \\
(16.5)\end{array}$ & $\begin{array}{c}0.965 \pm 0.003 \\
(11.0)\end{array}$ & $\begin{array}{c}0.963 \pm 0.003 \\
(22.0)\end{array}$ & $\begin{array}{c}0.964 \pm 0.003 \\
(16.5)\end{array}$ & $\begin{array}{c}0.963 \pm 0.003 \\
(22.0)\end{array}$ & $\begin{array}{c}0.963 \pm 0.002 \\
(22.0)\end{array}$ \\
\hline 4 & $\begin{array}{c}0.962 \pm 0.005 \\
(4.5)\end{array}$ & $\begin{array}{c}0.963 \pm 0.003 \\
(1.5)\end{array}$ & $\begin{array}{c}0.960 \pm 0.005 \\
(9.5)\end{array}$ & $\begin{array}{c}0.962 \pm 0.006 \\
(4.5)\end{array}$ & $\begin{array}{c}0.961 \pm 0.004 \\
(7.5)\end{array}$ & $\begin{array}{c}0.914 \pm 0.007 \\
(15.0)\end{array}$ \\
\hline 5 & $0.762 \pm 0.021$ & $\begin{array}{c}0.759 \pm 0.020 \\
(6.5)\end{array}$ & $\begin{array}{c}0.764 \pm 0.017 \\
(2.0)\end{array}$ & $\begin{array}{c}0.758 \pm 0.019 \\
(8.0)\end{array}$ & $\begin{array}{c}0.772 \pm 0.022 \\
(1.0)\end{array}$ & $\begin{array}{c}0.714 \pm 0.015 \\
(19.5)\end{array}$ \\
\hline 6 & $\begin{array}{c}0.544 \pm 0.014 \\
(11.0)\end{array}$ & $\begin{array}{c}0.547 \pm 0.013 \\
(4.0)\end{array}$ & $\begin{array}{c}0.549 \pm 0.014 \\
(3.0)\end{array}$ & $\begin{array}{c}0.540 \pm 0.014 \\
(16.0)\end{array}$ & $\begin{array}{c}0.550 \pm 0.013 \\
(1.5)\end{array}$ & $0.546 \pm 0.014$ \\
\hline 7 & $\begin{array}{c}0.765 \pm 0.006 \\
(21.0)\end{array}$ & $\begin{array}{c}0.765 \pm 0.007 \\
(21.0)\end{array}$ & $\begin{array}{c}0.764 \pm 0.006 \\
(23.0)\end{array}$ & $\begin{array}{c}0.763 \pm 0.009 \\
(24.0)\end{array}$ & $\begin{array}{c}0.768 \pm 0.008 \\
(15.0)\end{array}$ & $\begin{array}{c}0.768 \pm 0.006 \\
(15.0)\end{array}$ \\
\hline 8 & $\begin{array}{c}0.647 \pm 0.018 \\
(3.5)\end{array}$ & $\begin{array}{c}0.646 \pm 0.017 \\
(5.5)\end{array}$ & $\begin{array}{c}0.648 \pm 0.015 \\
(2.0)\end{array}$ & $\begin{array}{c}0.633 \pm 0.013 \\
\quad(10.0)\end{array}$ & $\begin{array}{c}0.649 \pm 0.017 \\
(1.0)\end{array}$ & $\begin{array}{c}0.616 \pm 0.015 \\
(13.0)\end{array}$ \\
\hline 9 & $\begin{array}{c}0.851 \pm 0.009 \\
(7.5)\end{array}$ & $\begin{array}{c}0.850 \pm 0.008 \\
(10.5)\end{array}$ & $\begin{array}{c}0.846 \pm 0.008 \\
(18.0)\end{array}$ & $0.852 \pm 0.007$ & $\begin{array}{c}0.847 \pm 0.006 \\
(16.0)\end{array}$ & $\begin{array}{c}0.857 \pm 0.005 \\
(1.0)\end{array}$ \\
\hline 10 & $\begin{array}{c}0.655 \pm 0.019 \\
(4.0)\end{array}$ & $\begin{array}{c}0.653 \pm 0.018 \\
(6.5)\end{array}$ & $\begin{array}{c}0.647 \pm 0.018 \\
(19.0)\end{array}$ & $\begin{array}{c}0.652 \pm 0.019 \\
(8.0)\end{array}$ & $\begin{array}{c}0.647 \pm 0.016 \\
(19.0)\end{array}$ & $\begin{array}{c}0.653 \pm 0.020 \\
(6.5)\end{array}$ \\
\hline 11 & $\begin{array}{c}0.710 \pm 0.011 \\
(19.5)\end{array}$ & $\begin{array}{c}0.711 \pm 0.011 \\
(17.0)\end{array}$ & $\begin{array}{c}0.724 \pm 0.010 \\
(8.5)\end{array}$ & $\begin{array}{c}0.708 \pm 0.011 \\
(23.0)\end{array}$ & $\begin{array}{c}0.724 \pm 0.013 \\
(8.5)\end{array}$ & $\begin{array}{c}0.741 \pm 0.008 \\
(1.0)\end{array}$ \\
\hline 12 & $\begin{array}{c}0.800 \pm 0.011 \\
(8.0)\end{array}$ & $\begin{array}{c}0.801 \pm 0.013 \\
(5.0)\end{array}$ & $\begin{array}{c}0.798 \pm 0.016 \\
(10.5)\end{array}$ & $\begin{array}{c}0.805 \pm 0.017 \\
(1.0)\end{array}$ & $\begin{array}{c}0.800 \pm 0.014 \\
(8.0)\end{array}$ & $\begin{array}{c}0.772 \pm 0.017 \\
(16.0)\end{array}$ \\
\hline 13 & $\begin{array}{c}0.916 \pm 0.003 \\
(4.5)\end{array}$ & $\begin{array}{c}0.916 \pm 0.003 \\
(4.5)\end{array}$ & $\begin{array}{c}0.914 \pm 0.003 \\
(12.5)\end{array}$ & $\begin{array}{c}0.916 \pm 0.003 \\
(4.5)\end{array}$ & $\begin{array}{c}0.915 \pm 0.003 \\
(8.5)\end{array}$ & $\begin{array}{c}0.914 \pm 0.003 \\
(12.5)\end{array}$ \\
\hline 14 & $\begin{array}{c}0.876 \pm 0.015 \\
(7.0)\end{array}$ & $\begin{array}{c}0.881 \pm 0.012 \\
(3.0)\end{array}$ & $0.879 \pm 0.012$ & $\begin{array}{c}0.875 \pm 0.012 \\
(8.0)\end{array}$ & $\begin{array}{c}0.880 \pm 0.013 \\
(4.0)\end{array}$ & $\begin{array}{c}0.676 \pm 0.007 \\
(16.0)\end{array}$ \\
\hline 15 & $\begin{array}{c}0.936 \pm 0.011 \\
(17.0)\end{array}$ & $\begin{array}{c}0.936 \pm 0.013 \\
(17.0)\end{array}$ & $\begin{array}{c}0.934 \pm 0.013 \\
(23.5)\end{array}$ & $\begin{array}{c}0.936 \pm 0.011 \\
(17.0)\end{array}$ & $\begin{array}{c}0.934 \pm 0.014 \\
\quad(23.5)\end{array}$ & $\begin{array}{c}0.965 \pm 0.008 \\
(1.0)\end{array}$ \\
\hline 16 & $0.880 \pm 0.016$ & $0.883 \pm 0.011$ & $0.881 \pm 0.011$ & $\begin{array}{c}0.877 \pm 0.013 \\
(9.0)\end{array}$ & $\begin{array}{c}0.882 \pm 0.011 \\
(2.5)\end{array}$ & $\begin{array}{c}0.678 \pm 0.006 \\
(16.0)\end{array}$ \\
\hline 17 & $\begin{array}{c}0.838 \pm 0.004 \\
(10.0)\end{array}$ & $\begin{array}{c}0.837 \pm 0.004 \\
(15.5)\end{array}$ & $\begin{array}{c}0.838 \pm 0.004 \\
(10.0)\end{array}$ & $\begin{array}{c}0.837 \pm 0.003 \\
(15.5)\end{array}$ & $\begin{array}{c}0.837 \pm 0.003 \\
(15.5)\end{array}$ & $\begin{array}{c}0.842 \pm 0.003 \\
(4.0)\end{array}$ \\
\hline 18 & $\begin{array}{c}0.738 \pm 0.022 \\
(7.5)\end{array}$ & $\begin{array}{c}0.749 \pm 0.018 \\
(4.5)\end{array}$ & $\begin{array}{c}0.759 \pm 0.015 \\
(2.0)\end{array}$ & $\begin{array}{c}0.738 \pm 0.020 \\
(7.5)\end{array}$ & $\begin{array}{c}0.757 \pm 0.016 \\
(3.0)\end{array}$ & $\begin{array}{c}0.565 \pm 0.000 \\
(17.5)\end{array}$ \\
\hline 19 & $\begin{array}{c}0.940 \pm 0.003 \\
(17.5)\end{array}$ & $\begin{array}{c}0.940 \pm 0.003 \\
(17.5)\end{array}$ & $\begin{array}{c}0.941 \pm 0.003 \\
(10.0)\end{array}$ & $\begin{array}{c}0.940 \pm 0.003 \\
(17.5)\end{array}$ & $\begin{array}{c}0.941 \pm 0.003 \\
(10.0)\end{array}$ & $\begin{array}{c}0.939 \pm 0.003 \\
(22.5)\end{array}$ \\
\hline 20 & $\begin{array}{c}0.937 \pm 0.006 \\
(13.5)\end{array}$ & $\begin{array}{c}0.936 \pm 0.005 \\
(16.0)\end{array}$ & $\begin{array}{c}0.935 \pm 0.006 \\
(20.5)\end{array}$ & $\begin{array}{c}0.935 \pm 0.006 \\
(20.5)\end{array}$ & $\begin{array}{c}0.935 \pm 0.006 \\
(20.5)\end{array}$ & $\begin{array}{c}0.937 \pm 0.005 \\
(13.5)\end{array}$ \\
\hline 21 & $\begin{array}{c}0.871 \pm 0.017 \\
(12.5)\end{array}$ & $\begin{array}{c}0.871 \pm 0.016 \\
(12.5)\end{array}$ & $\begin{array}{c}0.872 \pm 0.017 \\
(10.5)\end{array}$ & $\begin{array}{c}0.868 \pm 0.017 \\
(16.5)\end{array}$ & $\begin{array}{c}0.875 \pm 0.016 \\
(9.0)\end{array}$ & $\begin{array}{c}0.895 \pm 0.019 \\
(3.5)\end{array}$ \\
\hline 22 & $\begin{array}{c}0.763 \pm 0.007 \\
(15.5)\end{array}$ & $\begin{array}{c}0.765 \pm 0.008 \\
(9.5)\end{array}$ & $\begin{array}{c}0.764 \pm 0.008 \\
(13.5)\end{array}$ & $\begin{array}{c}0.764 \pm 0.007 \\
(13.5)\end{array}$ & $\begin{array}{c}0.765 \pm 0.008 \\
(9.5)\end{array}$ & $\begin{array}{c}0.766 \pm 0.006 \\
(4.0)\end{array}$ \\
\hline 23 & $\begin{array}{c}0.756 \pm 0.023 \\
(5.0)\end{array}$ & $\begin{array}{c}0.755 \pm 0.030 \\
(6.0)\end{array}$ & $\begin{array}{c}0.766 \pm 0.024 \\
(1.0)\end{array}$ & $\begin{array}{c}0.746 \pm 0.026 \\
(9.0)\end{array}$ & $\begin{array}{c}0.758 \pm 0.027 \\
(3.0)\end{array}$ & $\begin{array}{c}0.563 \pm 0.002 \\
(16.0)\end{array}$ \\
\hline 24 & $\begin{array}{c}0.754 \pm 0.017 \\
\quad(22.0)\end{array}$ & $\begin{array}{c}0.761 \pm 0.020 \\
(18.0)\end{array}$ & $\begin{array}{c}0.773 \pm 0.017 \\
(15.5)\end{array}$ & $\begin{array}{c}0.753 \pm 0.015 \\
\quad(23.0)\end{array}$ & $\begin{array}{c}0.768 \pm 0.016 \\
(17.0)\end{array}$ & $\begin{array}{c}0.794 \pm 0.000 \\
(7.0)\end{array}$ \\
\hline 25 & $\begin{array}{c}0.715 \pm 0.011 \\
(11.0)\end{array}$ & $\begin{array}{c}0.715 \pm 0.010 \\
(11.0)\end{array}$ & $\begin{array}{c}0.715 \pm 0.009 \\
(11.0)\end{array}$ & $\begin{array}{c}0.706 \pm 0.008 \\
(15.0)\end{array}$ & $\begin{array}{c}0.718 \pm 0.011 \\
(7.0)\end{array}$ & $0.721 \pm 0.007$ \\
\hline 26 & $\begin{array}{c}0.780 \pm 0.014 \\
(3.0)\end{array}$ & $\begin{array}{c}0.780 \pm 0.012 \\
(3.0)\end{array}$ & $\begin{array}{c}0.778 \pm 0.013 \\
(8.0)\end{array}$ & $\begin{array}{c}0.774 \pm 0.013 \\
(14.0)\end{array}$ & $\begin{array}{c}0.779 \pm 0.013 \\
(5.5)\end{array}$ & $\begin{array}{c}0.776 \pm 0.011 \\
(12.5)\end{array}$ \\
\hline 27 & $0.974 \pm 0.009$ & $0.974 \pm 0.011$ & $\begin{array}{c}0.977 \pm 0.009 \\
(1.0)\end{array}$ & $\begin{array}{c}0.972 \pm 0.010 \\
(7.5)\end{array}$ & $\begin{array}{c}0.970 \pm 0.011 \\
(10.0)\end{array}$ & $\begin{array}{c}0.940 \pm 0.012 \\
(16.0)\end{array}$ \\
\hline 28 & $\begin{array}{c}0.597 \pm 0.007 \\
(10.5)\end{array}$ & $\begin{array}{c}0.596 \pm 0.006 \\
(14.5)\end{array}$ & $\begin{array}{c}0.596 \pm 0.005 \\
(14.5)\end{array}$ & $\begin{array}{c}0.598 \pm 0.006 \\
(7.5)\end{array}$ & $\begin{array}{c}0.596 \pm 0.007 \\
(14.5)\end{array}$ & $\begin{array}{c}0.599 \pm 0.006 \\
(4.0)\end{array}$ \\
\hline 29 & $\begin{array}{c}0.529 \pm 0.015 \\
(18.5)\end{array}$ & $\begin{array}{c}0.532 \pm 0.016 \\
(10.0)\end{array}$ & $\begin{array}{c}0.532 \pm 0.015 \\
\quad(10.0)\end{array}$ & $\begin{array}{c}0.532 \pm 0.012 \\
(10.0)\end{array}$ & $\begin{array}{c}0.532 \pm 0.012 \\
\quad(10.0)\end{array}$ & $\begin{array}{c}0.531 \pm 0.013 \\
(14.5)\end{array}$ \\
\hline 30 & $\begin{array}{c}0.597 \pm 0.005 \\
(6.5) \\
\end{array}$ & $\begin{array}{c}0.597 \pm 0.005 \\
(6.5) \\
\end{array}$ & $\begin{array}{c}0.597 \pm 0.005 \\
(6.5) \\
\end{array}$ & $\begin{array}{c}0.597 \pm 0.005 \\
(6.5) \\
\end{array}$ & $\begin{array}{c}0.598 \pm 0.005 \\
(1.5) \\
\end{array}$ & $\begin{array}{c}0.594 \pm 0.004 \\
(17.0) \\
\end{array}$ \\
\hline Rank & 11.217 & 10.017 & $\begin{array}{c}10.800 \\
\end{array}$ & $\begin{array}{l}12.700 \\
\end{array}$ & $\begin{array}{c}10.233 \\
\end{array}$ & $\begin{array}{c}10.583 \\
\end{array}$ \\
\hline
\end{tabular}

is the hidden-layer output matrix for testing instances, $g(z)=\frac{1}{1+e^{-z}}$ is sigmoid activation function, 


$$
W_{D \times L}=\left[\begin{array}{llll}
\mathrm{w}_{1} & \mathrm{w}_{2} & \cdots & \mathrm{w}_{L}
\end{array}\right]=\left[\begin{array}{cccc}
w_{11} & w_{21} & \cdots & w_{L 1} \\
w_{12} & w_{22} & \cdots & w_{L 2} \\
\vdots & \vdots & \ddots & \vdots \\
w_{1 D} & w_{2 D} & \cdots & w_{L D}
\end{array}\right] \text { and } \mathrm{b}=\left[\begin{array}{c}
b_{1} \\
b_{2} \\
\vdots \\
b_{L}
\end{array}\right]
$$

are input-layer weight and hidden-layer biases which are usually determined by assigning the random numbers obeying uniform distribution. Our main work in this paper is to use the probability distributions in Table 1 to initialize $W_{D \times L}$ and b and compare the classification performances of corresponding ELMs.

\section{Impact of Random Initialization on ELM's Generalization Performance}

\subsection{Data Preparation Guideline}

In this experimental study, 30 UCI data sets are used to test the classification performances of ELMs corresponding to different random initializations. The detailed description of 30 data sets is summarized in Table 2. In order to use these data sets more efficiently and specifically, we preprocess them according to the following guidelines: (1) Delete nominal-value attributes. ELMs are mainly used to handle the classification problem with continuous-value attributes. Up to now, we don't find the study regarding how to use ELM to deal with nominal-value attributes. (2) Replace missing attribute-values. The unsupervised filter named ReplaceMissingValues in Weka [18] is used to replace all missing attribute-values in each data set. (3) Reduce large data sets. The unsupervised filter named Resample with the sampleSizePercent 10 in Weka is used to randomly reduce the sizes of three large data sets: Magic Telescope, Page Blocks and Wine Quality-White.

\subsection{Experimental Setup}

We use 10 -fold cross-validation to get the training and testing accuracies corresponding to 24 ELMs (i.e., initializing the input-layer weights and hidden-layer biases with random numbers obeying 24 different probability distributions in Table 1 respectively) on every data set. In order to make the experimental results more reliable, 10 -fold cross-validation on each data set is repeated 100 times for every ELM and the average values of 100 training and testing accuracies are used as the finally experimental results. In every time of 10 -fold cross-validation, the different ELMs are trained on the same training sets and evaluated on the same testing set and the random initializations to input-layer weights and hidden-layer biases are independent.

The number of hidden-layer nodes in all ELMs used in our experimental study is 50. The necessary parameters in 24 probability distributions are initialized as follows: (1) $\alpha=2$ and $\beta=2$ for Beta; (2) $\lambda=1$ for Cauchy; (3) $k=2$ for Central Chi-Squared; (4) $k=2$ for Chi; (5) $\lambda=1$ and $k=2$ for Erlang; (6) $\lambda=1$ for Exponential; (7) $n_{1}=5$ and $n_{2}=2$ for F; (8) $s=1$ and $\alpha=3$ Frechet; (9) $k=9$ and $\theta=0.1$ Gamma; (10) $\mu=1$ and $\beta=2$ Gumbel; (11) no parameter for Hyperbolic Secant; (12) $\mu=0$ and $b=1$ for Laplace; (13) $\mu=9$ and $s=3$ for Logistic; (14) $\mu=0$ and $\sigma=1$ Lognormal; (15) $\mu=0$ and $\sigma=1$ Normal; (16) $x_{m}=1$ and $\alpha=3$ Pareto; (17) $\sigma=0.5$ for Rayleigh; (18) $r=1$ for Semicircle; (19) $v=5$ for Student's-t; (20) $a=-2.5, c=0$ and $b=2.5$ for Triangular; (21) $a=-1, b=1, \mu=0$ and $\sigma=1$ for Truncated Normal; (22) $a=-2.5$ and $b=2.5$ for Uniform; (23) $\mu=0$ and $k=4$ for Von Mises; and (24) $\lambda=1$ and $k=5$ for Weibull.

\subsection{Experimental Result and Analysis}

The training and testing accuracies of 24 ELMs on 30 UCI data sets are summarized in Tables 3 and 4 respectively. Here, we use Wilcoxon signed-ranks test and Friedman test [3] to conduct the statistical analysis to above-mentioned experimental results. The former is to assess whether the classification 
accuracies of two ELMs differ on a single data set, while the latter is to test whether or not the classification accuracies of these 24 ELMs are equal on all data sets. Here, we only give the detailed procedures of these two statistical tests based on training accuracies of different ELMs. Similarly, the testing accuracies can be dealt with.

The compared objects of Wilcoxon signed-ranks test are the respective 100 training accuracies of two ELMs $\left(\mathrm{ELM}_{1}\right.$ and $\left.\mathrm{ELM}_{2}\right)$ on a single data set.

1. Let $a_{i}^{(1)}$ and $a_{i}^{(2)}$ denote the training accuracies of ELM ${ }_{1}$ and $\operatorname{ELM}_{2}$ in the $i$-th $(i=1,2, \cdots, 100)$ run of 10 -fold cross-validation respectively.

2. $d_{i}=a_{i}^{(1)}-a_{i}^{(2)}, i=1,2, \cdots, 100$ denotes the difference between the training accuracies of ELM 1 and ELM 2 in the $i$-th run of 10 -fold cross-validation.

3. Increasingly order $\left|d_{i}\right|, i=1,2, \cdots, 100$ starting with the smallest as 1 . For $d_{i}$ with same absolute values, the average of their ranks is assigned as the new rank. Let $\operatorname{rank}\left(d_{i}\right)$ denote the rank of $d_{i}$.

4. Let $R_{+}$and $R_{-}$denote the sums of ranks for the runs on which ELM ${ }_{1}$ is better and worse than ELM 2 respectively. $R_{+}$and $R_{-}$can be calculated as follows:

$$
R_{+}=\sum_{\substack{i=1 \\ d_{i}>0}}^{100} \operatorname{rank}\left(d_{i}\right)+\frac{1}{2} \sum_{\substack{i=1 \\ d_{i}=0}}^{100} \operatorname{rank}\left(d_{i}\right)
$$

and

$$
R_{-}=\sum_{\substack{i=1 \\ d_{i}<0}}^{100} \operatorname{rank}\left(d_{i}\right)+\frac{1}{2} \sum_{\substack{i=1 \\ d_{i}=0}}^{100} \operatorname{rank}\left(d_{i}\right)
$$

5. Construct the statistic $z=\frac{\min \left(R_{+}, R_{-}\right)-\frac{1}{4} m(m+1)}{\sqrt{\frac{1}{24} m(m+1)(2 m+1)}}$ based on the null-hypothesis, which indicates that ELM1 and ELM2 have the equal training accuracy on a given data set, $m$ is the times of 10 -fold cross-validations. In our study, $m=100$. When $m \geq 30$, the distribution of $z$ is approximately normal.

6. Set the significance level $\alpha=0.1$. If $z>z_{\frac{\alpha}{2}}$ or $z<-z_{\frac{\alpha}{2}}$, the null-hypothesis can be rejected, i.e., there are some differences between $\mathrm{ELM}_{1}$ and $\mathrm{ELM}_{2}$; else, we accept the null-hypothesis. The value $z_{\frac{0.1}{2}}=z_{0.05}=1.645$ can be found from any statistical textbook.

After we compare the training accuracies of two ELMs on a single data set, Friedman test is used to analyze the difference of training accuracies corresponding to 24 ELMs on 30 data sets. The analyzed object is the experimental results in Table 3.

1. Increasingly order the average training accuracies of 24 ELMs on every data set. Then, the average value of ranks is assigned to the same training accuracy (This depends on the results obtained by previous Wilcoxon signed-ranks test: if the null-hypothesis is accepted, we say the training accuracies of two ELMs are same and thus have the same rank). In Table 3, the number in parentheses is the rank of training accuracy.

2. For 24 ELMs, the average value of $\operatorname{ranks} R_{i}, i=1,2, \cdots, 24$ on 30 data sets is calculated respectively (i.e., the last line of Table 3).

3. Construct the statistic $F_{F}=\frac{(m-1) \chi_{F}^{2}}{m(k-1)-\chi_{F}^{2}}$ based on the null-hypothesis which indicates that all models have the equal training accuracy on all data sets, where $\chi_{F}^{2}=\frac{12 m}{k(k+1)}\left[\sum_{j=1}^{k} R_{j}^{2}-\frac{k(k+1)^{2}}{4}\right]$ is the Friedman statistic, $k$ is the number of learning models, $m$ is the number of data sets. In this study, $k=24$ and $m=30$. 
4. The statistic $F_{F}$ obeys the F-distribution with $(k-1)$ and $(k-1) \times(m-1)$ degrees of freedom. Under the significance level $\alpha=0.1$, we can obtain

$$
F_{\alpha}((k-1),(k-1) \times(m-1))=F_{0.1}(23,667)=1.403 .
$$

5. If $F_{F}>F_{0.1}(23,667)$, the null-hypothesis can be rejected. That is to say the training accuracies of these 24 ELMs are different on the 30 data sets.

Based on the analyzed results with Wilcoxon signed-ranks test and Friedman test, we increasingly order these 24 probability distributions in Fig. 1 according to the average ranks of training and testing accuracies respectively. From Fig. 1, we give the following interestingly experimental observations and usefully empirical conclusions.

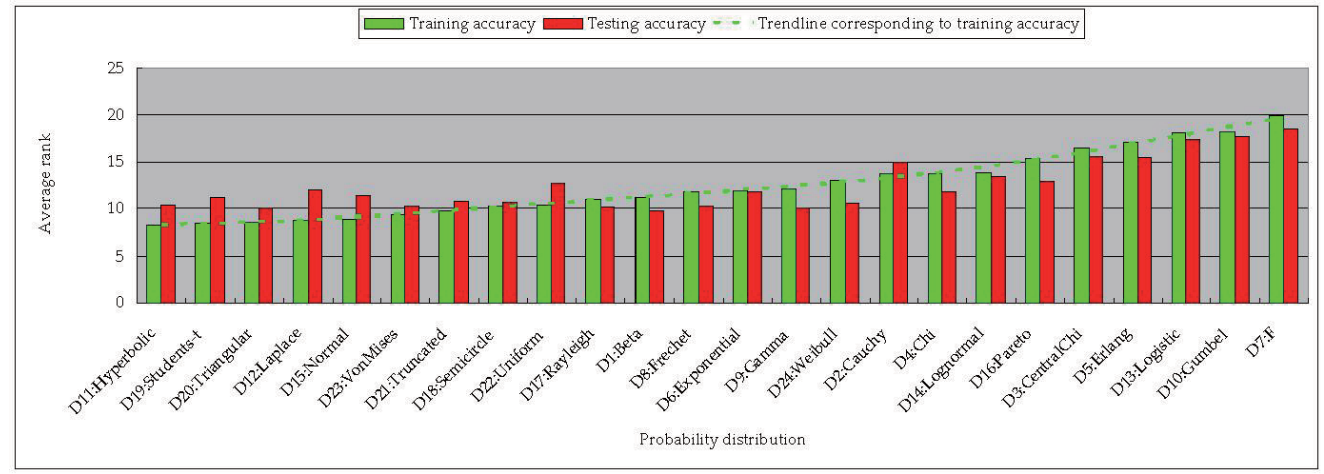

(a). Training Accuracy

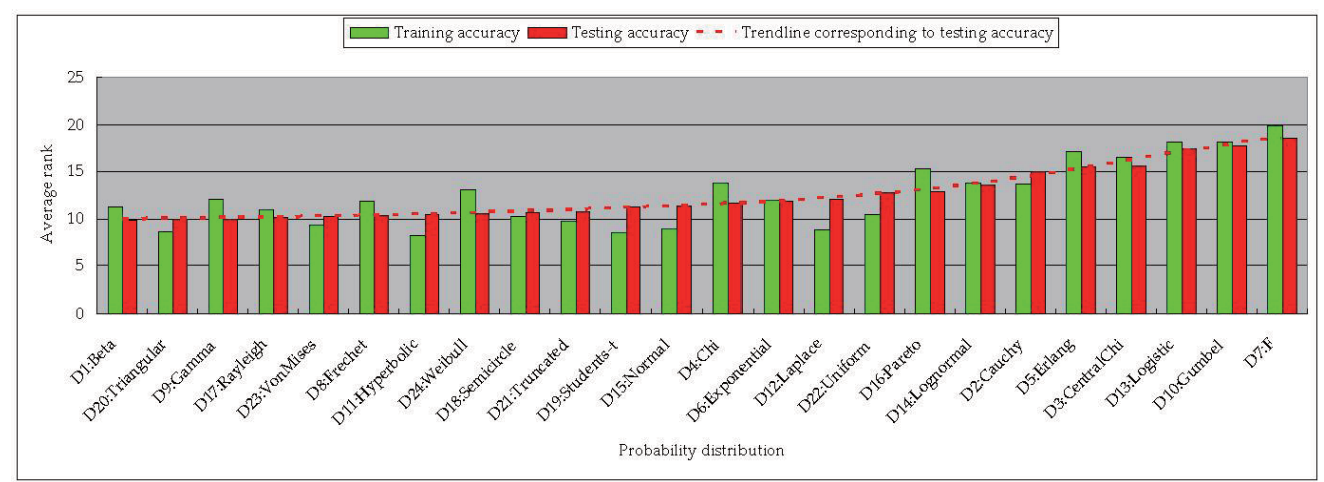

(b). Testing Accuracy

Fig. 1. Increasingly order these 24 probability distributions according to the average ranks of training and testing.

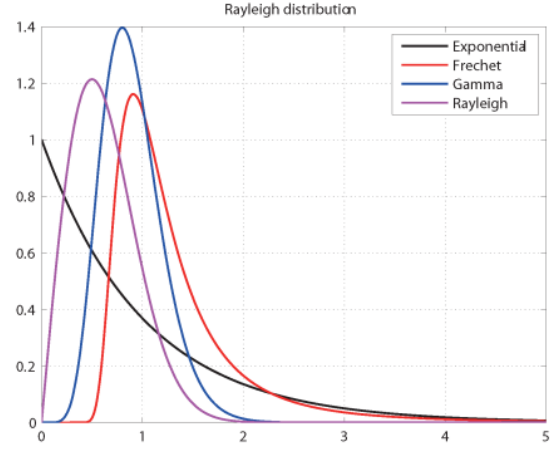

(a) Heavy-tailed distributions

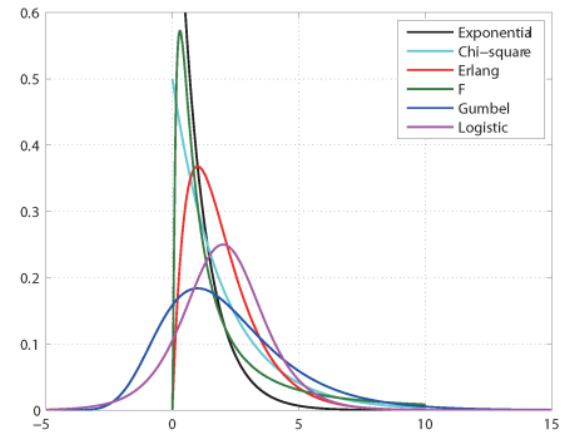

(b) Light-tailed distributions

Fig. 2. Heavy-tailed and light-tailed distributions for ELM initialization. 
1. ELMs initialized with Hyperbolic Secant, Student's-t, Laplace and Normal distributions obtain the better training accuracies. Meanwhile, these four distributions are more easily to cause the over-fitting of ELM. We can see that the orders of training accuracies corresponding to these four distributions are 1, 2, 4, and 5 respectively in Fig. 1-(a), while their orders of testing accuracies in Fig. 1-(b) are 7, 11, 15 and 12. That is to say Hyperbolic Secant, Student's-t, Laplace and Normal distributions make ELM obtain higher training accuracy and lower testing accuracies. Some common characteristics of these probability distributions are (1) their probability density functions $p(x)$ are bell-shaped; (2) all $p(x)$ are symmetrical with respect to $x=0$; and (3) the support intervals of $p(x)$ are $x \in(-\infty,+\infty)$.

2. ELMs initialized with Gamma, Rayleigh and Frechet distributions obtain the better testing accuracies. We can see that the orders of testing accuracies corresponding to these three distributions are 3,4 , and 6 respectively in Fig. 1-(b), while their orders of training accuracies in Fig. 1-(a) are 14, 10 and 12. This indicates that Gamma, Rayleigh and Frechet distributions make ELM have the better predictive capability to unseen instances. The common characteristics of these probability distributions include (1) their probability density functions $p(x)$ have the heavy tails; (2) $p(x)$ are asymmetrical with respect to $x=0$; and (3) the support intervals of $p(x)$ are $x \in(0,+\infty)$.

3. The mostly-used Uniform distribution cannot bring about the better testing accuracy for ELM. The order of testing accuracy of Uniform distribution is 16 in Fig. 1-(b). The distributions with light tail, e.g., central Chi-Squared, Erlang, F, Gumbel and Logistic are unsuited to initialize the input-layer weights and hidden-layer biases for ELM, because they lead to the worse training and testing accuracies on selected 30 data sets.

In Fig. 2, we plot the probability density functions for heavy-tailed Gamma, Rayleigh and Frechet in Fig. 2-(a) and light-tailed (Central Chi-Squared, Erlang, F, Gumbel and Logistic in Fig. 2-(b)) distributions. An intuitive explanation to heavy-tailed distribution is its probability density function $p_{1}(x)$ has a heavier tail than Exponential distribution, i.e., the right tail of distribution $p_{1}(x)$ will be located below Exponential distribution with the increase of $x$. In contrast with heavy-tailed distribution, the light-tailed distribution $p_{2}(x)$ has a lighter tail than Exponential distribution, the right tail of distribution $p_{2}(x)$ will be located above Exponential distribution with the increase of $x$. From Fig. 1, we can see that the heavy-tailed distributions (e.g., (Gamma, Rayleigh and Frechet) obtain the better generalization performances than Uniform distribution and the light-tailed distributions (e.g., Central Chi-Squared, Erlang, F, Gumbel and Logistic) get the worse training and testing accuracies than Uniform distribution.

\section{Conclusion}

In this paper, we experimentally validate the impact of different continuous probability distributions on the classification accuracies of ELMs and give some important and useful enlightenments regarding ELM initialization. By initializing the input-layer weights and hidden-layer biases with random numbers obeying 24 different continuous probability distributions, we compare the training and testing accuracies of 24 ELMs on 30 UCI data sets. The experimental results and statistical analysis reflect that (1) the symmetrical and bell-shaped probability distributions have the higher training accuracies and meanwhile easily cause the over-fitting of ELM; (2) the heavy-tailed distributions bring about the higher testing accuracies for ELM than Uniform distribution; and (3) the light-tailed distributions obtain the worse training and testing accuracies than Uniform distribution.

\section{Acknowledgement}

We thank Editor-in-Chief and anonymous reviewers for their valuable comments and suggestions which help us to improve this paper significantly. This paper was supported by National Natural Science 
Foundation of China (61503252) and China Postdoctoral Science Foundation (2016T90799).

\section{References}

[1] Huang, G. B., Zhu, Q. Y., \& Siew, C. K. (2006). Extreme learning machine: Theory and applications. Neurocomputing, 70(1), 489-501.

[2] Serre, D. (2006). Matrices: Theory and Applications. New York: Springer.

[3] Huang, G. B., Chen, L., \& Siew C. K. (2006). Universal approximation using incremental constructive feedforward networks with random hidden nodes. IEEE Transactions on Neural Networks, 17(4), 879-892.

[4] Huang, G. B., Wang, D. H., \& Lan, Y. (2011). Extreme learning machines: A survey. International Journal of Machine Learning and Cybernetics, 2(2), 107-122.

[5] Zhu, Q. Y., Qin, A., Suganthan, P., \& Huang, G. B. (2005). Evolutionary extreme learning machine. Pattern Recognition, 38(10), 1759-1763.

[6] Cao, J., Lin, Z., \& Huang, G. B. (2012). Self-adaptive evolutionary extreme learning machine. Neural Processing Letters, 36(3), 285-305.

[7] Matias, T., Souza, F., Araújo, R., \& Antunes, C. H. (2014). Learning of a single-hidden layer feedforward neural network using an optimized extreme learning machine. Neurocomputing, 129, 428-436.

[8] Heeswijk, M. V., \& Miche, Y. (2015). Binary/ternary extreme learning machines. Neurocomputing, 149, 187-197.

[9] Balasundaram, S., \& Gupta, D. (2014). On optimization based extreme learning machine in primal for regression and classification by functional iterative method. International Journal of Machine Learning and Cybernetics.

[10] Deng, W., Zheng, Q., \& Chen, L. (2009). Regularized extreme learning machine. Proceedings of 2009 IEEE Symposium on Computational Intelligence and Data Mining (PP. 389-395).

[11] Horata, P., Chiewchanwattana, S., \& Sunat, K. (2013). Robust extreme learning machine. Neurocomputing, 102, 31-44.

[12] Huang, G. B., Li, M. B., Chen, L., \& Siew, C. K. (2008). Incremental extreme learning machine with fully complex hidden nodes. Neurocomputing, 71(4-6), 576-583.

[13] Miche, Y., Sorjamaa, A., Bas, P., Simula, O., Jutten, C., \& Lendasse, A. (2010). OP-ELM: Optimally pruned extreme learning machine. IEEE Transactions on Neural Networks, 21(1), 158-162.

[14] Wang, X. Z., Shao, Q. Y., Miao, Q., \& Zhai, J. H. (2013). Architecture selection for networks trained with extreme learning machine using localized generalization error model. Neurocomputing, 102, 3-9.

[15] Zhang, K., \& Luo, M. X. (2015). Outlier-robust extreme learning machine for regression problems. Neurocomputing, 151, 1519-1527.

[16] He, Y. L., Liu, J. N. K., Wang, X. Z., \& Hu, Y. X. (2012). Optimal bandwidth selection for resubstitution entropy estimation. Applied Mathematics and Computation, 219(8), 3425-3460.

[17] Demšar, J. (2006). Statistical comparisons of classifiers over multiple data sets. Journal of Machine Learning Research, 7(1), 1-30.

[18] Hall, M., Frank, E., Holmes, G., Pfahringer, B., Reutemann, P., \& Witten, I. H. (2009). The WEKA data mining software: An update. SIGKDD Explorations, 11(1), 10-18.

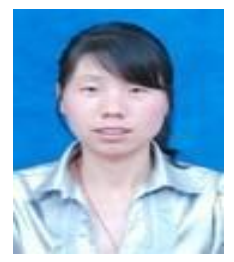

Xiaofang Zhang completed her bachelor and master degree in computer science from Wuhan University of Science and Technology, Wuhan China. From 2008 to 201, she worked as an assistant in Fujian Normal University Minnan Science and Technology Institute. From 2011 to 2016, she worked as a lecturer in Beijing Jiaotong University Haibin College. Since 
2017, she is working as a lecturer in City College, Wuhan University of Science and Technology. Her main research interests include machine learning and big data mining.

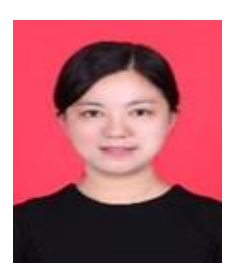

Xiaoli Lin received the B.S and M.S degree from Wuhan University of Science and Technology of China, Hubei, China, in 2004 and 2007. She is currently a doctoral student in the College of Computer Science and Technology, Wuhan University of Science and Technology, China. She is mainly interested in computational biology and bioinformatics.

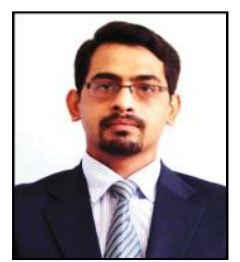

Rana Aamir Raza Ashfaq completed his Ph.D from Shenzhen University, Shenzhen China. He recieved his master degree in computer science from Blekinge Tekniska Hgskola (BTH), Sweden. He also received his bachelor and master degrees in computer science from Bahauddin Zakariya University, Multan, Pakistan. Since 2010 he is working as assistant professor in Department of Computer Science, Bahauddin Zakariya University, Multan, Pakistan. His main research interests include machine learning and big data mining. 